\title{
A hybrid iterative method for a common solution of variational inequalities, generalized mixed equilibrium problems, and hierarchical fixed point problems
}

Hui-ying Hu and Lu-Chuan Ceng*

"Correspondence:

zenglc@hotmail.com Department of Mathematics,

Shanghai Normal University,

Shanghai, 200234, China

\begin{abstract}
In this paper, we suggest and analyze an iterative method for finding a common solution of variational inequalities, a generalized mixed equilibrium problem, and a hierarchical fixed point problem in the setting of a real Hilbert space. Under suitable conditions, we prove the strong convergence theorem. Several special cases are also discussed. The results presented in this paper extend and improve some well-known results in the literature.
\end{abstract}

MSC: 49J30; 47H09; 47H2O

Keywords: variational inequalities; generalized mixed equilibrium problems; hierarchical fixed point problems; fixed point problems

\section{Introduction}

Let $H$ be a real Hilbert space whose inner product and norm are denoted by $\langle\cdot, \cdot\rangle$ and $\|\cdot\|$. Let $C$ be a nonempty, closed, and convex subset of $H$. Let $F: C \times C \rightarrow R$ be a bifunction, $D: C \rightarrow H$ be a nonlinear mapping, and $\varphi: C \rightarrow R$ be a function. Recently, Peng and Yao [1] considered the generalized mixed equilibrium problem (GMEP) which involves finding $x \in C$ such that

$$
F(x, y)+\varphi(y)-\varphi(x)+\langle D x, y-x\rangle \geq 0, \quad \forall y \in C .
$$

The set of solutions of $(1.1)$ is denoted by $\operatorname{GMEP}(F, \varphi, D)$. The GMEP is very general in the sense that it includes, as special cases, optimization problems, variational inequalities, minimax problems, and Nash equilibrium problems; see, for example, [2-5]. For instance, we refer to [6] for a general system generalized equilibrium problems.

If $D=0$, then the generalized mixed equilibrium problem (GMEP) (1.1) becomes the following mixed equilibrium problem (MEP): Find $x \in C$ such that

$$
F(x, y)+\varphi(y)-\varphi(x) \geq 0, \quad \forall y \in C .
$$

Problem (1.2) was studied by Ceng and Yao [7]. The set of solutions of (1.2) is denoted by $\operatorname{MEP}(F, \varphi)$.

(c) $2015 \mathrm{Hu}$ and Ceng; licensee Springer. This article is distributed under the terms of the Creative Commons Attribution 4.0 International License (http://creativecommons.org/licenses/by/4.0/), which permits unrestricted use, distribution, and reproduction in any medium, provided you give appropriate credit to the original author(s) and the source, provide a link to the Creative Commons license, and indicate if changes were made. 
If $\varphi=0$, then the generalized mixed equilibrium problem (GMEP) (1.1) becomes the following generalized equilibrium problem (GEP): Find $x \in C$ such that

$$
F(x, y)+\langle D x, y-x\rangle \geq 0, \quad \forall y \in C .
$$

Problem (1.3) was studied by Takahashi and Takahashi [8]. The set of solutions of (1.3) is denoted by $\operatorname{GEP}(F, D)$.

If $\varphi=0$ and $D=0$, then the generalized mixed equilibrium problem (GMEP) (1.1) becomes the following equilibrium problem (EP): Find $x \in C$ such that

$$
F(x, y) \geq 0, \quad \forall y \in C
$$

The solution set of (1.4) is denoted by $E P(F)$. Numerous problems in physics, optimization, and economics reduce to finding a solution of $(1.4)$; see $[9,10]$.

Let $A: C \rightarrow H$, and let $F(x, y)=\langle A x, y-x\rangle, \forall x, y \in C$. Then $x \in E P(F)$ if and only if $\langle A x, y-x\rangle \geq 0, \forall y \in C$, which is a classical variational inequality problem (VIP): Find a vector $u \in C$ such that

$$
\langle v-u, A u\rangle, \quad \forall v \in C
$$

The solution set of (1.5) is denoted by $V I(C, A)$. It is easy to observe that

$$
u^{*} \in V I(C, A) \quad \Longleftrightarrow \quad u^{*}=P_{C}\left[u^{*}-\rho A u^{*}\right], \quad \text { where } \rho>0 .
$$

We now have a variety of techniques to suggest and analyze various iterative algorithms for solving variational inequalities and related optimization problems; see [1-31]. The fixed point theory has played an important role in the development of various algorithms for solving variational inequalities. Using the projection operator technique, one usually establishes an equivalence between variational inequalities and fixed point problems. We introduce the following definitions, which are useful in the following analysis.

Definition 1.1 The mapping $T: C \rightarrow H$ is said to be

(a) monotone if

$$
\langle T x-T y, x-y\rangle \geq 0, \quad \forall x, y \in C ;
$$

(b) strongly monotone if there exists $\alpha>0$ such that

$$
\langle T x-T y, x-y\rangle \geq \alpha\|x-y\|^{2}, \quad \forall x, y \in C ;
$$

(c) $\alpha$-inverse strongly monotone if there exists $\alpha>0$ such that

$$
\langle T x-T y, x-y\rangle \geq \alpha\|T x-T y\|^{2}, \quad \forall x, y \in C ;
$$

(d) nonexpansive if

$$
\|T x-T y\| \leq\|x-y\|, \quad \forall x, y \in C ;
$$


(e) $k$-Lipschitz continuous if there exists a constant $k>0$ such that

$$
\|T x-T y\| \leq k\|x-y\|, \quad \forall x, y \in C ;
$$

(f) a contraction on $C$ if there exists a constant $0 \leq k \leq 1$ such that

$$
\|T x-T y\| \leq k\|x-y\|, \quad \forall x, y \in C .
$$

It is easy to observe that every $\alpha$-inverse strongly monotone $T$ is monotone and Lipschitz continuous. It is well known that every nonexpansive operator $T: H \rightarrow H$ satisfies, for all $(x, y) \in H \times H$, the inequality

$$
\langle(x-T x)-(y-T y), T y-T x\rangle \leq \frac{1}{2}\|(T x-x)-(T y-y)\|^{2},
$$

and therefore, we get, for all $(x, y) \in H \times \operatorname{Fix}(T)$,

$$
\langle x-T x, y-T x\rangle \leq \frac{1}{2}\|T x-x\|^{2} .
$$

The fixed point problem for the mapping $T$ is to find $x \in C$ such that

$$
T x=x .
$$

We denote by $F(T)$ the set of solutions of (1.8). It is well known that $F(T)$ is closed and convex, and $P_{F}(T)$ is well defined.

Recently, many researchers studied various iterative algorithms for finding an element of $V I(C, A) \cap F(S)$. Takahashi and Toyoda [11] introduced the following iterative scheme:

$$
x_{n+1}=\alpha_{n} x_{n}+\left(1-\alpha_{n}\right) S P_{C}\left(I-\lambda_{n} B\right) x_{n}, \quad \forall n \geq 0 .
$$

They proved that the sequence $\left\{x_{n}\right\}$ converges weakly to a point $q \in V I(C, B) \cap F(S)$. Yao and Yao [12] introduced the following scheme:

$$
\left\{\begin{array}{l}
x_{1}=u \in C, \\
y_{n}=P_{C}\left(I-\lambda_{n} A\right) x_{n}, \\
x_{n+1}=\alpha_{n} u+\beta_{n} x_{n}+\gamma_{n} S P_{C}\left(I-\lambda_{n} A\right) y_{n},
\end{array}\right.
$$

and obtain some convergence theorems. Later, Chang et al. [9] introduced the following iterative scheme:

$$
\left\{\begin{array}{l}
\phi\left(u_{n}, y\right)+\frac{1}{r_{n}}\left\langle y-u_{n}, u_{n}-x_{n}\right\rangle \geq 0, \quad \forall y \in C, \\
x_{n+1}=\alpha_{n} f\left(x_{n}\right)+\beta_{n} x_{n}+\gamma_{n} W_{n} k_{n}, \\
k_{n}=P_{C}\left(I-\lambda_{n} B\right) y_{n}, \\
y_{n}=P_{C}\left(I-\lambda_{n} B\right) u_{n},
\end{array}\right.
$$


and obtained some convergence theorems. In 2014, Zhou et al. [13] introduced the following iterative scheme:

$$
\left\{\begin{array}{l}
F\left(y_{n}, \eta\right)+\left\langle D y_{n}, \eta-y_{n}\right\rangle+\frac{1}{r_{n}}\left\langle\eta-y_{n}, y_{n}-x_{n}\right\rangle \geq 0, \quad \forall \eta \in C, \\
\rho_{n}=\sum_{m=1}^{r} \eta_{n}^{m} P_{C}\left(I-\mu_{m} B_{m}\right) y_{n} \\
x_{n+1}=\alpha_{n} \gamma f\left(x_{n}\right)+\beta_{n} x_{n}+\left(\left(1-\beta_{n}\right) I-\alpha_{n} A\right) W_{n} \rho_{n}
\end{array}\right.
$$

where $A$ is a strongly positive bounded linear operator, $f$ is a contraction on $H$, and $W_{n}$ is the $W$-mapping of $C$ into itself which is generated by a family of nonexpansive mappings $S_{n}, S_{n-1}, \ldots, S_{1}$, and a sequence of positive numbers in $[0,1], \lambda_{n}, \lambda_{n-1}, \ldots, \lambda_{1}$, then they obtained some strong convergence theorems.

On the other hand, let $S: C \rightarrow H$ be a nonexpansive mapping. The following problem is called a hierarchical fixed point problem (in short, HFPP): Find $x \in F(T)$ such that

$$
\langle x-S x, y-x\rangle \geq 0, \quad \forall y \in F(T)
$$

It is well known that the hierarchical fixed point problem (1.13) links with some monotone variational inequalities and convex programming problems; see [14]. Various methods have been proposed to solve the hierarchical fixed point problem; see [15-19]. In 2010, Yao et al. [14] introduced the following strong convergence iterative algorithm to solve problem (1.13):

$$
\left\{\begin{array}{l}
y_{n}=\beta_{n} S x_{n}+\left(1-\beta_{n}\right) x_{n}, \\
x_{n+1}=P_{C}\left[\alpha_{n} f\left(x_{n}\right)+\left(1-\alpha_{n}\right) T y_{n}\right], \quad \forall n \geq 0
\end{array}\right.
$$

where $f: C \rightarrow H$ is a contraction mapping and $\left\{\alpha_{n}\right\},\left\{\beta_{n}\right\}$ are two sequences in $(0,1)$. Under certain restrictions on the parameters, Yao et al. proved that the sequence $\left\{x_{n}\right\}$ generated by (1.14) converges strongly to $z \in F(T)$, which is the unique solution of the following variational inequality:

$$
\langle(I-f) z, y-z\rangle \geq 0, \quad \forall y \in F(T) .
$$

In 2011, Ceng et al. [20] investigated the following iterative method:

$$
x_{n+1}=P_{C}\left[\alpha_{n} \rho U\left(x_{n}\right)+\left(I-\alpha_{n} \mu F\right) T\left(y_{n}\right)\right], \quad \forall n \geq 0,
$$

where $U$ is a Lipschitzian mapping, and $F$ is a Lipschitzian and strongly monotone mapping. They proved that under some assumptions as regards approximations on the operators and parameters, the sequence generated by (1.16) converges strongly to the unique solution of the variational inequality

$$
\langle\rho U(z)-\mu F(z), x-z\rangle \leq 0, \quad \forall x \in F(T)
$$

Very recently, Ceng et al. [21] introduced and analyzed hybrid implicit and explicit viscosity iterative algorithms for solving a general system of variational inequalities with a hierarchical fixed point problem constraint for a countable family of nonexpansive mapping in a real Banach space, which can be viewed as an extension and improvement of the 
recent results in the literature. In 2014, Bnouhachem et al. [22] introduced the following iterative method:

$$
\left\{\begin{array}{l}
F\left(u_{n}, y\right)+\frac{1}{r_{n}}\left\langle y-u_{n}, u_{n}-x_{n}\right\rangle \geq 0, \quad \forall y \in C ; \\
y_{n}=\beta_{n} S x_{n}+\left(1-\beta_{n}\right) u_{n} ; \\
x_{n+1}=P_{C}\left[\alpha_{n} \rho U\left(x_{n}\right)+\gamma_{n} x_{n}+\left(\left(1-\gamma_{n}\right) I-\alpha_{n} \mu F\right) T\left(y_{n}\right)\right], \quad \forall n \geq 0,
\end{array}\right.
$$

where $U$ and $F$ are the same as above. They proved that under some assumptions as regards approximations on the operators and parameters, the sequence $\left\{x_{n}\right\}$ generated by (1.17) converges strongly to the unique solution of the variational inequality

$$
\langle\rho U(z)-\mu F(z), x-z\rangle \leq 0, \quad \forall x \in F(T) \cap E P(F) .
$$

In the same year, Bnouhachem and Chen [23] introduced the following iterative method:

$$
\left\{\begin{array}{l}
F\left(u_{n}, y\right)+\left\langle D x_{n}, y-u_{n}\right\rangle+\varphi(y)-\varphi\left(u_{n}\right)+\frac{1}{r_{n}}\left\langle y-u_{n}, u_{n}-x_{n}\right\rangle \geq 0, \quad \forall y \in C ; \\
z_{n}=P_{C}\left[u_{n}-\lambda_{n} A u_{n}\right] ; \\
y_{n}=\beta_{n} S x_{n}+\left(1-\beta_{n}\right) z_{n} ; \\
x_{n+1}=P_{C}\left[\alpha_{n} \rho U\left(x_{n}\right)+\gamma_{n} x_{n}+\left(\left(1-\gamma_{n}\right) I-\alpha_{n} \mu F\right) T\left(y_{n}\right)\right], \quad \forall n \geq 0,
\end{array}\right.
$$

where $U$ and $F$ are the same as above. They proved that under some assumptions as regards approximations on the operators and parameters, the sequence $\left\{x_{n}\right\}$ generated by (1.18) converges strongly to the unique solution of variational inequality

$$
\langle\rho U(z)-\mu F(z), x-z\rangle \leq 0, \quad \forall x \in V I(C, A) \cap \operatorname{GMEP}(F, \varphi, D) \cap F(T) .
$$

In this paper, motivated by the work of Zhou et al. [13], Bnouhachem et al. [22, 23] and others, we give an iterative method for finding the approximate element of the common set of solutions of GMEP (1.1), VIP (1.5) and HFPP (1.13) in real Hilbert space. We establish a strong convergence theorem for the sequence generated by the proposed method. The proposed method is quite general and flexible and includes several well-known methods for solving variational inequality problems, mixed equilibrium problems, and hierarchical fixed point problems; see, e.g., [6, 13, 22-27] and the references therein.

\section{Preliminaries}

In this section, we list some fundamental lemmas that are useful in the consequent analysis. The first lemma provides some basic properties of the projection onto $C$.

Lemma 2.1 Let $P_{C}$ denote the projection of $H$ onto $C$. Then we have the following inequalities:

$$
\begin{aligned}
& \left\langle z-P_{C}[z], P_{C}[z]-v\right\rangle \geq 0, \quad \forall z \in H, v \in C ; \\
& \left\langle u-v, P_{C}[u]-P_{C}[v]\right\rangle \geq\left\|P_{C}[u]-P_{C}[v]\right\|^{2}, \quad \forall u, v \in H ; \\
& \left\|P_{C}[u]-P_{C}[v]\right\| \leq\|u-v\|, \quad \forall u, v \in H ; \\
& \left\|u-P_{C}[z]\right\|^{2} \leq\|z-u\|^{2}-\left\|z-P_{C}[z]\right\|^{2}, \quad \forall z \in H, u \in C .
\end{aligned}
$$


Assumption 2.1 [1] Let $F: C \times C \rightarrow R$ be a bifunction and $\varphi: C \rightarrow R$ be a function satisfying the following assumptions:

$\left(\mathrm{A}_{1}\right) F(x, x)=0, \forall x \in C$;

$\left(\mathrm{A}_{2}\right) F$ is monotone, i.e., $F(x, y)+F(y, x) \leq 0, \forall x, y \in C$;

$\left(\mathrm{A}_{3}\right)$ for each $x, y, z \in C, \lim _{t \rightarrow 0} F(t z+(1-t) x, y) \leq F(x, y)$;

$\left(\mathrm{A}_{4}\right)$ for each $x \in C, y \rightarrow F(x, y)$ is convex and lower semicontinuous;

$\left(\mathrm{B}_{1}\right)$ for each $x \in H$ and $r>0$, there exists a bounded sunset $K$ of $C$ and $y_{x} \in C \cap \operatorname{dom}(\varphi)$ such that

$$
F\left(z, y_{x}\right)+\varphi\left(y_{x}\right)-\varphi(z)+\frac{1}{r}\left\langle y_{x}-z, z-x\right\rangle \leq 0, \quad \forall z \in C \backslash K
$$

$\left(\mathrm{B}_{2}\right) \quad C$ is a bounded set.

Lemma 2.2 [1] Let $C$ be a nonempty, closed, and convex subset of $H$. Let $F: C \times C \rightarrow R$ satisfy $\left(\mathrm{A}_{1}\right)-\left(\mathrm{A}_{4}\right)$, and let $\varphi: C \rightarrow R$ be a proper lower semicontinuous and convex function. Assume that either $\left(\mathrm{B}_{1}\right)$ or $\left(\mathrm{B}_{2}\right)$ holds. For $r>0$ and $\forall x \in H$, define a mapping $T_{r}: H \rightarrow C$ as follows:

$$
T_{r}(x)=\left\{z \in C: F(z, y)+\varphi(y)+\varphi(z)+\frac{1}{r}\langle y-z, z-x\rangle \geq 0, \forall y \in C\right\} .
$$

Then the following hold:

(i) $T_{r}$ is nonempty and single-valued;

(ii) $T_{r}$ is firmly nonexpansive, i.e.,

$$
\left\|T_{r}(x)-T_{r}(y)\right\|^{2} \leq\left\langle T_{r}(x)-T_{r}(y), x-y\right\rangle, \quad \forall x, y \in H
$$

(iii) $F\left(T_{r}(I-r D)\right)=\operatorname{GMEP}(F, \varphi, D)$;

(iv) $\operatorname{GMEP}(F, \varphi, D)$ is closed and convex.

Lemma 2.3 [28] (Demiclosedness principle) Let $T: C \rightarrow C$ be a nonexpansive mapping with $\operatorname{Fix}(T) \neq \emptyset$. If $\left\{x_{n}\right\}$ is a sequence in $C$ that converges weakly to $x$ and if $\left\{(I-T) x_{n}\right\}$ converges strongly to $y$, then $(I-T) x=y$; in particular, if $y=0$, then $x \in \operatorname{Fix}(T)$.

Lemma 2.4 [20] Let $U: C \rightarrow H$ be a $\tau$-Lipschitzian mapping, and let $F: C \rightarrow H$ be a $\kappa$ Lipschitzian and $\eta$-strongly monotone mapping, then for $0 \leq \rho \tau<\mu \eta, \mu F-\rho U$ is $\mu \eta-\rho \tau$ strongly monotone, i.e.,

$$
\langle(\mu F-\rho U) x-(\mu F-\rho U) y, x-y\rangle \geq(\mu \eta-\rho \tau)\|x-y\|^{2}, \quad \forall x, y \in C .
$$

Lemma 2.5 [29] Suppose that $\lambda \in(0,1)$ and $\mu>0$. Let $F: C \rightarrow H$ be a $\kappa$-Lipschitzian and $\eta$-strongly monotone operator. In association with a nonexpansive mapping $T: C \rightarrow C$, define the mapping $T^{\lambda}: C \rightarrow H$ by

$$
T^{\lambda} x=T x-\lambda \mu F T(x), \quad \forall x \in C .
$$


Then $T^{\lambda}$ is a contraction provided $\mu<\frac{2 \eta}{\kappa^{2}}$, that is,

$$
\left\|T^{\lambda} x-T^{\lambda} y\right\| \leq(1-\lambda \nu)\|x-y\|, \quad \forall x, y \in C,
$$

where $v=1-\sqrt{1-\mu\left(2 \eta-\mu \kappa^{2}\right)}$.

Lemma 2.6 [30] Let $\left\{s_{n}\right\}$ be a sequence of non-negative real numbers satisfying

$$
s_{n+1} \leq\left(1-\omega_{n}\right) s_{n}+\omega_{n} \delta_{n}+\gamma_{n}, \quad \forall n \geq 0,
$$

where $\left\{\omega_{n}\right\},\left\{\delta_{n}\right\}$, and $\left\{\gamma_{n}\right\}$ satisfying the following conditions:

(i) $\left\{\omega_{n}\right\} \subset[0,1]$ and $\sum_{n=0}^{\infty} \omega_{n}=\infty$,

(ii) $\lim \sup _{n \rightarrow \infty} \delta_{n} \leq 0$ or $\sum_{n=0}^{\infty} \omega_{n}\left|\delta_{n}\right|<\infty$,

(iii) $\gamma_{n} \geq 0(n \geq 0), \sum_{n=0}^{\infty} \gamma_{n}<\infty$.

Then $\lim _{n \rightarrow \infty} s_{n}=0$.

Lemma 2.7 [31] Let $C$ be a closed convex subset of a real Hilbert H. Let $\left\{T_{m}: 1 \leq m \leq r\right\}$ be a sequence of nonexpansive mappings on $C$. Suppose that $\bigcap_{m=1}^{r} F\left(T_{m}\right)$ is nonempty. Let $\left\{\lambda_{m}\right\}$ be a sequence of positive numbers with $\sum_{m=1}^{r} \lambda_{m}=1$. Then a mapping $S$ on $C$ defined by

$$
S x=\sum_{m=1}^{r} \lambda_{m} T_{m} x
$$

for all $x \in C$ is well defined, nonexpansive, and $F(S)=\bigcap_{m=1}^{r} F\left(T_{m}\right)$ holds.

\section{Main result}

In this section, we suggest and analyze our method for finding common solutions of the generalized mixed equilibrium problem (1.1), the variational problem (1.5), and the hierarchical fixed point problem (1.13).

Let $C$ be a nonempty, closed, and convex subset of a real Hilbert space $H$. Let $B_{m}: C \rightarrow H$ be a $l_{m}$-inverse strongly monotone mapping for each $1 \leq m \leq r$, where $r$ is some positive integer. Let $D: C \rightarrow H$ be a $\theta$-inverse strongly monotone mapping. Let $F: C \times C \rightarrow R$ satisfy $\left(\mathrm{A}_{1}\right)-\left(\mathrm{A}_{4}\right)$, and let $\varphi: C \rightarrow R$ be a proper lower semicontinuous and convex function. Let $S, T: C \rightarrow C$ be nonexpansive mappings and such that $\mathscr{F}=F(T) \cap V I\left(C, B_{m}\right) \cap$ $\operatorname{GMEP}(F, \varphi, D) \neq \emptyset$. Let $F: C \rightarrow H$ be a $\kappa$-Lipschitzian mapping and $\eta$-strongly monotone, and let $U: C \rightarrow H$ be a $\tau$-Lipschitzian mapping.

Algorithm 3.1 For an arbitrary given $x_{0} \in C$, let the iterative sequences $\left\{u_{n}\right\},\left\{v_{n}\right\}$, $\left\{x_{n}\right\}$, and $\left\{y_{n}\right\}$ be generated by

$$
\left\{\begin{array}{l}
F\left(u_{n}, y\right)+\left\langle D x_{n}, y-u_{n}\right\rangle+\varphi(y)-\varphi\left(u_{n}\right)+\frac{1}{r_{n}}\left\langle y-u_{n}, u_{n}-x_{n}\right\rangle \geq 0, \quad \forall y \in C ; \\
v_{n}=\delta_{n} u_{n}+\left(1-\delta_{n}\right) \sum_{m=1}^{r} \eta_{n}^{m} P_{C}\left(I-\mu_{m} B_{m}\right) u_{n} ; \\
y_{n}=\beta_{n} S x_{n}+\left(1-\beta_{n}\right) v_{n} ; \\
x_{n+1}=P_{C}\left[\alpha_{n} \rho U\left(x_{n}\right)+\gamma_{n} x_{n}+\left(\left(1-\gamma_{n}\right) I-\alpha_{n} \mu F\right) T\left(y_{n}\right)\right], \quad \forall n \geq 0,
\end{array}\right.
$$


where $\mu_{m} \in\left(0,2 l_{m}\right),\left\{r_{n}\right\} \subset(0,2 \theta)$. Suppose that the parameters satisfy $0<\mu<\frac{2 \eta}{\kappa^{2}}, 0 \leq$ $\rho \tau<v$, where $v=1-\sqrt{1-\mu\left(2 \eta-\mu \kappa^{2}\right)}$. Also $\left\{\alpha_{n}\right\},\left\{\beta_{n}\right\},\left\{\gamma_{n}\right\}$, and $\left\{\delta_{n}\right\}$ are sequences in $(0,1)$ satisfying the following conditions:

(a) $\lim _{n \rightarrow \infty} \alpha_{n}=0$, and $\sum_{n=1}^{\infty} \alpha_{n}=\infty$;

(b) $\lim _{n \rightarrow \infty}\left(\beta_{n} / \alpha_{n}\right)=0$;

(c) $\lim _{n \rightarrow \infty} \gamma_{n}=0$, and $\gamma_{n}+\alpha_{n}<1$;

(d) $\sum_{n=1}^{\infty}\left|\alpha_{n}-\alpha_{n-1}\right|<\infty, \sum_{n=1}^{\infty}\left|\beta_{n}-\beta_{n-1}\right|<\infty, \sum_{n=1}^{\infty}\left|\gamma_{n}-\gamma_{n-1}\right|<\infty$, and $\sum_{n=1}^{\infty}\left|\delta_{n}-\delta_{n-1}\right|<\infty$

(e) $\liminf _{n \rightarrow \infty} r_{n}>0$, and $\sum_{n=1}^{\infty}\left|r_{n}-r_{n-1}\right|<\infty$;

(f) $\lim _{n \rightarrow \infty} \eta_{n}^{m}=\eta^{m} \in(0,1)$ for each $m$, where $1 \leq m \leq r$;

(g) $\sum_{m=1}^{r} \eta_{n}^{m}=1, \forall n \geq 1$.

Remark 3.1 Our method can be reviewed as an extension and improvement for some well-known results, for example, the following:

(i) The (self-)contraction mapping $f: H \rightarrow H$ in [13], Theorem 10 is extended to the case of a Lipschitzian (possibly nonself-)mapping $U: C \rightarrow H$ on a nonempty, closed, and convex subset $C$ of $H$.

(ii) The strongly positive linear bounded operator $A$ in [13], Theorem 10 is extended to the case of the $\kappa$-Lipschitzian mapping and $\eta$-strongly monotone (possibly nonself-)mapping $F: C \rightarrow H$.

(iii) The contractive coefficient $h \in(0,1)$ in [13], Theorem 10 is extended to the case where the Lipschitzian constant $\tau$ lies in $[0, \infty)$.

(iv) The equilibrium problem in [13], Theorem 10 is extended to the case of the generalized mixed equilibrium problem.

(v) If $D=\varphi=0, B_{m}=0$ for each $m$, and $\delta_{n}=0$, then the proposed method is an extension and improvement of a method studied in [22].

(vi) If $\delta_{n}=0, m=1$, then we obtain an extension and improvement of a method in [23].

(vii) If $\rho=\mu=1, \beta_{n}=\delta_{n}=0, \varphi=0, U=f$ a contraction mapping, $F=A$ a strongly positive linear bounded operator, and $T=W_{n}$, where $W_{n}$ is the $W$-mapping of $C$ into itself which is generated by a family of nonexpansive mappings $S_{n}, S_{n-1}, \ldots, S_{1}$, and a sequence of positive numbers in $[0,1] \lambda_{n}, \lambda_{n-1}, \ldots, \lambda_{1}$, then the proposed method is an extension and improvement of a method studied in [13].

This shows that Algorithm 3.1 is quite general and unifying.

Lemma 3.1 Let $x^{*} \in \mathscr{F}=F(T) \cap V I\left(C, B_{m}\right) \cap \operatorname{GMEP}(F, \varphi, D)$. Then $\left\{x_{n}\right\},\left\{u_{n}\right\}$, $\left\{v_{n}\right\}$, and $\left\{y_{n}\right\}$ are bounded.

Proof First, we show that the mapping $I-r_{n} D$ is nonexpansive. For any $x, y \in C$,

$$
\begin{aligned}
\left\|\left(I-r_{n} D\right) x-\left(I-r_{n} D\right) y\right\|^{2} & =\left\|(x-y)-r_{n}(D x-D y)\right\|^{2} \\
& =\|x-y\|^{2}-2 r_{n}\langle x-y, D x-D y\rangle+r_{n}^{2}\|D x-D y\|^{2} \\
& \leq\|x-y\|^{2}-r_{n}\left(2 \theta-r_{n}\right)\|D x-D y\|^{2} \\
& \leq\|x-y\|^{2} .
\end{aligned}
$$


Similarly, we can show that the mapping $I-\mu_{m} B_{m}$ is nonexpansive for each $1 \leq m \leq r$. For each $1 \leq m \leq r$, put

$$
w_{n}^{m}=P_{C}\left(I-\mu_{m} B_{m}\right) u_{n} \quad \text { and } \quad z_{n}=\sum_{m=1}^{r}\left(\eta_{n}^{m} w_{n}^{m}\right) .
$$

Then Algorithm 3.1 can be rewritten as

$$
\left\{\begin{array}{l}
F\left(u_{n}, y\right)+\left\langle D x_{n}, y-u_{n}\right\rangle+\varphi(y)-\varphi\left(u_{n}\right)+\frac{1}{r_{n}}\left\langle y-u_{n}, u_{n}-x_{n}\right\rangle \geq 0, \quad \forall y \in C ; \\
v_{n}=\delta_{n} u_{n}+\left(1-\delta_{n}\right) z_{n} ; \\
y_{n}=\beta_{n} S x_{n}+\left(1-\beta_{n}\right) v_{n} ; \\
x_{n+1}=P_{C}\left[\alpha_{n} \rho U\left(x_{n}\right)+\gamma_{n} x_{n}+\left(\left(1-\gamma_{n}\right) I-\alpha_{n} \mu F\right) T\left(y_{n}\right)\right], \quad \forall n \geq 0 .
\end{array}\right.
$$

Fixing $x \in \mathscr{F}$, we have

$$
\begin{aligned}
\left\|w_{n}^{m}-x^{*}\right\| & =\left\|P_{C}\left(I-\mu_{m} B_{m}\right) u_{n}-P_{C}\left(I-\mu_{m} B_{m}\right) x^{*}\right\| \\
& \leq\left\|u_{n}-x^{*}\right\|, \quad 1 \leq \forall m \leq r .
\end{aligned}
$$

It follows that

$$
\begin{aligned}
\left\|z_{n}-x^{*}\right\| & =\left\|\sum_{m=1}^{r}\left(\eta_{n}^{m} w_{n}^{m}\right)-x^{*}\right\| \leq \sum_{m=1}^{r} \eta_{n}^{m}\left\|w_{n}^{m}-x^{*}\right\| \\
& \leq\left\|u_{n}-x^{*}\right\| .
\end{aligned}
$$

It follows from Lemma 2.2 that $u_{n}=T_{r_{n}}\left(x_{n}-r_{n} D x_{n}\right)$ and $x^{*}=T_{r_{n}}\left(x^{*}-r_{n} D x^{*}\right)$, we have

$$
\begin{aligned}
\left\|u_{n}-x^{*}\right\|^{2} & =\left\|T_{r_{n}}\left(x_{n}-r_{n} D x_{n}\right)-T_{r_{n}}\left(x^{*}-r_{n} D x^{*}\right)\right\|^{2} \\
& \leq\left\|\left(x_{n}-r_{n} D x_{n}\right)-\left(x^{*}-r_{n} D x^{*}\right)\right\|^{2} \\
& \leq\left\|x_{n}-x^{*}\right\|^{2}-r_{n}\left(2 \theta-r_{n}\right)\left\|D x_{n}-D x^{*}\right\|^{2} \\
& \leq\left\|x_{n}-x^{*}\right\|^{2} .
\end{aligned}
$$

From (3.1) and the above inequalities, we have

$$
\begin{aligned}
\left\|v_{n}-x^{*}\right\|^{2} & \leq \delta_{n}\left\|u_{n}-x^{*}\right\|^{2}+\left(1-\delta_{n}\right)\left\|z_{n}-x^{*}\right\|^{2} \\
& \leq \delta_{n}\left\|u_{n}-x^{*}\right\|^{2}+\left(1-\delta_{n}\right)\left\|u_{n}-x^{*}\right\|^{2} \\
& \leq\left\|u_{n}-x^{*}\right\|^{2} \\
& \leq\left\|x_{n}-x^{*}\right\|^{2}-r_{n}\left(2 \theta-r_{n}\right)\left\|D x_{n}-D x^{*}\right\|^{2} \\
& \leq\left\|x_{n}-x^{*}\right\| .
\end{aligned}
$$

Then we have

$$
\begin{aligned}
& \left\|z_{n}-x^{*}\right\|^{2} \leq\left\|u_{n}-x^{*}\right\|^{2} \leq\left\|x_{n}-x^{*}\right\|^{2}-r_{n}\left(2 \theta-r_{n}\right)\left\|D x_{n}-D x^{*}\right\|^{2} \leq\left\|x_{n}-x^{*}\right\|^{2}, \\
& \left\|v_{n}-x^{*}\right\|^{2} \leq\left\|u_{n}-x^{*}\right\|^{2} \leq\left\|x_{n}-x^{*}\right\|^{2}-r_{n}\left(2 \theta-r_{n}\right)\left\|D x_{n}-D x^{*}\right\|^{2} \leq\left\|x_{n}-x^{*}\right\|^{2} .
\end{aligned}
$$


We define $V_{n}=\alpha_{n} \rho U\left(x_{n}\right)+\gamma_{n} x_{n}+\left(\left(1-\gamma_{n}\right) I-\alpha_{n} \mu F\right) T\left(y_{n}\right)$. Next, we prove that the sequence $\left\{x_{n}\right\}$ is bounded, and without loss of generality, we can assume that $\beta_{n} \leq \alpha_{n}$ for all $n \geq 0$. From (3.1), we have

$$
\begin{aligned}
& \left\|x_{n+1}-x^{*}\right\| \\
& =\left\|P_{C}\left[V_{n}\right]-P_{C}\left[x^{*}\right]\right\| \\
& \leq\left\|\alpha_{n} \rho U\left(x_{n}\right)+\gamma_{n} x_{n}+\left(\left(1-\gamma_{n}\right) I-\alpha_{n} \mu F\right) T\left(y_{n}\right)-x^{*}\right\| \\
& =\| \alpha_{n}\left(\rho U\left(x_{n}\right)-\mu F\left(x^{*}\right)\right)+\gamma_{n}\left(x_{n}-x^{*}\right)+\left(\left(1-\gamma_{n}\right) I-\alpha_{n} \mu F\right) T\left(y_{n}\right) \\
& -\left(\left(1-\gamma_{n}\right) I-\alpha_{n} \mu F\right) T\left(x^{*}\right) \| \\
& \leq \alpha_{n}\left\|\rho U\left(x_{n}\right)-\mu F\left(x^{*}\right)\right\|+\gamma_{n}\left\|x_{n}-x^{*}\right\| \\
& +\left(1-\gamma_{n}\right)\left\|\left(I-\frac{\alpha_{n} \mu}{1-\gamma_{n}} F\right) T\left(y_{n}\right)-\left(I-\frac{\alpha_{n} \mu}{1-\gamma_{n}} F\right) T\left(x^{*}\right)\right\| \\
& =\alpha_{n}\left\|\rho U\left(x_{n}\right)-\rho U\left(x^{*}\right)+(\rho U-\mu F) x^{*}\right\|+\gamma_{n}\left\|x_{n}-x^{*}\right\| \\
& +\left(1-\gamma_{n}\right)\left\|\left(I-\frac{\alpha_{n} \mu}{1-\gamma_{n}} F\right) T\left(y_{n}\right)-\left(I-\frac{\alpha_{n} \mu}{1-\gamma_{n}} F\right) T\left(x^{*}\right)\right\| \\
& \leq \alpha_{n} \rho \tau\left\|x_{n}-x^{*}\right\|+\alpha_{n}\left\|(\rho U-\mu F) x^{*}\right\|+\gamma_{n}\left\|x_{n}-x^{*}\right\| \\
& +\left(1-\gamma_{n}\right)\left(1-\frac{\alpha_{n} v}{1-\gamma_{n}}\right)\left\|y_{n}-x^{*}\right\| \\
& \leq \alpha_{n} \rho \tau\left\|x_{n}-x^{*}\right\|+\alpha_{n}\left\|(\rho U-\mu F) x^{*}\right\|+\gamma_{n}\left\|x_{n}-x^{*}\right\| \\
& +\left(1-\gamma_{n}-\alpha_{n} v\right)\left\|\beta_{n} S x_{n}+\left(1-\beta_{n}\right) v_{n}-x^{*}\right\| \\
& \leq \alpha_{n} \rho \tau\left\|x_{n}-x^{*}\right\|+\alpha_{n}\left\|(\rho U-\mu F) x^{*}\right\|+\gamma_{n}\left\|x_{n}-x^{*}\right\| \\
& +\left(1-\gamma_{n}-\alpha_{n} v\right)\left(\beta_{n}\left\|S x_{n}-S x^{*}\right\|+\beta_{n}\left\|S x^{*}-x^{*}\right\|+\left(1-\beta_{n}\right)\left\|v_{n}-x^{*}\right\|\right) \\
& \leq \alpha_{n} \rho \tau\left\|x_{n}-x^{*}\right\|+\alpha_{n}\left\|(\rho U-\mu F) x^{*}\right\|+\gamma_{n}\left\|x_{n}-x^{*}\right\| \\
& +\left(1-\gamma_{n}-\alpha_{n} v\right)\left(\beta_{n}\left\|S x_{n}-S x^{*}\right\|+\beta_{n}\left\|S x^{*}-x^{*}\right\|+\left(1-\beta_{n}\right)\left\|x_{n}-x^{*}\right\|\right) \\
& \leq \alpha_{n} \rho \tau\left\|x_{n}-x^{*}\right\|+\alpha_{n}\left\|(\rho U-\mu F) x^{*}\right\|+\gamma_{n}\left\|x_{n}-x^{*}\right\| \\
& +\left(1-\gamma_{n}-\alpha_{n} v\right)\left(\beta_{n}\left\|x_{n}-x^{*}\right\|+\beta_{n}\left\|S x^{*}-x^{*}\right\|+\left(1-\beta_{n}\right)\left\|x_{n}-x^{*}\right\|\right) \\
& \leq\left(1-\alpha_{n}(v-\rho \tau)\right)\left\|x_{n}-x^{*}\right\|+\alpha_{n}\left\|(\rho U-\mu F) x^{*}\right\| \\
& +\left(1-\gamma_{n}-\alpha_{n} v\right) \beta_{n}\left\|S x^{*}-x^{*}\right\| \\
& \leq\left(1-\alpha_{n}(v-\rho \tau)\right)\left\|x_{n}-x^{*}\right\|+\alpha_{n}\left\|(\rho U-\mu F) x^{*}\right\|+\beta_{n}\left\|S x^{*}-x^{*}\right\| \\
& \leq\left(1-\alpha_{n}(v-\rho \tau)\right)\left\|x_{n}-x^{*}\right\|+\alpha_{n}\left(\left\|(\rho U-\mu F) x^{*}\right\|+\left\|S x^{*}-x^{*}\right\|\right) \\
& \leq\left(1-\alpha_{n}(v-\rho \tau)\right)\left\|x_{n}-x^{*}\right\|+\frac{\alpha_{n}(v-\rho \tau)}{v-\rho \tau}\left(\left\|(\rho U-\mu F) x^{*}\right\|+\left\|S x^{*}-x^{*}\right\|\right) \\
& \leq \max \left\{\left\|x_{n}-x^{*}\right\|, \frac{1}{v-\rho \tau}\left(\left\|(\rho U-\mu F) x^{*}\right\|+\left\|S x^{*}-x^{*}\right\|\right)\right\},
\end{aligned}
$$


where the third inequality follows from Lemma 2.5. By induction on $n$, we obtain $\| x_{n}-$ $x_{0} \| \leq \max \left\{\left\|x_{0}-x^{*}\right\|, \frac{1}{v-\rho \tau}\left(\left\|(\rho U-\mu F) x^{*}\right\|+\left\|S x^{*}-x^{*}\right\|\right)\right\}$ for $n \geq 0$ and $x_{0} \in C$. Hence $\left\{x_{n}\right\}$ is bounded, and, consequently, we deduce that $\left\{u_{n}\right\},\left\{v_{n}\right\}$, and $\left\{y_{n}\right\}$ are bounded.

Lemma 3.2 Let $x^{*} \in \mathscr{F}=F(T) \cap V I\left(C, B_{m}\right) \cap \operatorname{GMEP}(F, \varphi, D)$ and $\left\{x_{n}\right\}$ be generated by Algorithm 3.1. Then we have:

(a) $\lim _{n \rightarrow \infty}\left\|x_{n+1}-x_{n}\right\|=0$,

(b) the weak $w$-limit set $w_{w}\left(x_{n}\right) \subset F(T)\left(w_{w}\left(x_{n}\right)=\left\{x: x_{n_{i}}-x\right\}\right)$.

Proof Note that

$$
\begin{aligned}
\left\|w_{n}^{m}-w_{n-1}^{m}\right\| & =\left\|P_{C}\left(I-\mu_{m} B_{m}\right) u_{n}-P_{C}\left(I-\mu_{m} B_{m}\right) u_{n-1}\right\| \\
& \leq\left\|u_{n}-u_{n-1}\right\|, \quad 1 \leq \forall m \leq r .
\end{aligned}
$$

On the other hand, we have

$$
v_{n}-v_{n-1}=\delta_{n}\left(u_{n}-u_{n-1}\right)+\left(1-\delta_{n}\right)\left(z_{n}-z_{n-1}\right)+\left(\delta_{n}-\delta_{n-1}\right)\left(u_{n-1}-z_{n-1}\right) .
$$

It follows from (3.3) that

$$
\begin{aligned}
\| v_{n}- & v_{n-1} \| \\
\leq & \delta_{n}\left\|u_{n}-u_{n-1}\right\|+\left(1-\delta_{n}\right)\left\|z_{n}-z_{n-1}\right\|+\left|\delta_{n}-\delta_{n-1}\right|\left\|u_{n-1}-z_{n-1}\right\| \\
\leq & \delta_{n}\left\|u_{n}-u_{n-1}\right\|+\left(1-\delta_{n}\right)\left\|\sum_{m=1}^{r}\left(\eta_{n}^{m} w_{n}^{m}\right)-\sum_{m=1}^{r}\left(\eta_{n-1}^{m} w_{n-1}^{m}\right)\right\|+\left|\delta_{n}-\delta_{n-1}\right|\left\|u_{n-1}-z_{n-1}\right\| \\
\leq & \left(1-\delta_{n}\right)\left\|\sum_{m=1}^{r}\left(\eta_{n}^{m} w_{n}^{m}\right)-\sum_{m=1}^{r}\left(\eta_{n}^{m} w_{n-1}^{m}\right)+\sum_{m=1}^{r}\left(\eta_{n}^{m} w_{n-1}^{m}\right)-\sum_{m=1}^{r}\left(\eta_{n-1}^{m} w_{n-1}^{m}\right)\right\| \\
& +\delta_{n}\left\|u_{n}-u_{n-1}\right\|+\left|\delta_{n}-\delta_{n-1}\right|\left\|u_{n-1}-z_{n-1}\right\| \\
\leq & \left\|u_{n}-u_{n-1}\right\|+M \cdot \sum_{m=1}^{r}\left|\eta_{n}^{m}-\eta_{n-1}^{m}\right|+\left|\delta_{n}-\delta_{n-1}\right|\left\|u_{n-1}-z_{n-1}\right\|
\end{aligned}
$$

where $M=\max \left\{\sup \left\{\left\|P_{C}\left(I-\mu_{m} B_{m}\right) u_{n}\right\|: n \geq 1\right\}: 1 \leq m \leq r\right\}$. Next we estimate that

$$
\begin{aligned}
& \left\|y_{n}-y_{n-1}\right\| \\
& \quad \leq\left\|\beta_{n} S x_{n}+\left(1-\beta_{n}\right) v_{n}-\beta_{n-1} S x_{n-1}-\left(1-\beta_{n-1}\right) v_{n-1}\right\| \\
& \quad=\left\|\beta_{n}\left(S x_{n}-S x_{n-1}\right)+\left(1-\beta_{n}\right)\left(v_{n}-v_{n-1}\right)+\left(\beta_{n}-\beta_{n-1}\right)\left(S x_{n-1}-v_{n-1}\right)\right\| \\
& \quad \leq \beta_{n}\left\|x_{n}-x_{n-1}\right\|+\left(1-\beta_{n}\right)\left\|v_{n}-v_{n-1}\right\|+\left|\beta_{n}-\beta_{n-1}\right|\left(\left\|S x_{n-1}\right\|+\left\|v_{n-1}\right\|\right) .
\end{aligned}
$$

It follows from (3.4) and the above inequality that

$$
\begin{aligned}
\left\|y_{n}-y_{n-1}\right\| \leq & \beta_{n}\left\|x_{n}-x_{n-1}\right\|+\left(1-\beta_{n}\right)\left\{\left\|u_{n}-u_{n-1}\right\|+M \cdot \sum_{m=1}^{r}\left|\eta_{n}^{m}-\eta_{n-1}^{m}\right|\right. \\
& \left.+\left|\delta_{n}-\delta_{n-1}\right|\left\|u_{n-1}-z_{n-1}\right\|\right\}+\left|\beta_{n}-\beta_{n-1}\right|\left(\left\|S x_{n-1}\right\|+\left\|v_{n-1}\right\|\right) .
\end{aligned}
$$


On the other hand, $u_{n}=T_{r_{n}}\left(x_{n}-r_{n} D x_{n}\right)$ and $u_{n-1}=T_{r_{n-1}}\left(x_{n-1}-r_{n-1} D x_{n-1}\right)$, we have

$$
F\left(u_{n}, y\right)+\varphi(y)-\varphi\left(u_{n}\right)+\left\langle D x_{n}, y-u_{n}\right\rangle+\frac{1}{r_{n}}\left\langle y-u_{n}, u_{n}-x_{n}\right\rangle \geq 0, \quad \forall y \in C,
$$

and

$$
\begin{aligned}
& F\left(u_{n-1}, y\right)+\varphi(y)-\varphi\left(u_{n-1}\right)+\left\langle D x_{n-1}, y-u_{n-1}\right\rangle+\frac{1}{r_{n-1}}\left\langle y-u_{n-1}, u_{n-1}-x_{n-1}\right\rangle \geq 0, \\
& \quad \forall y \in C
\end{aligned}
$$

Taking $y=u_{n-1}$ in (3.6) and $y=u_{n}$ in (3.7), we get

$$
F\left(u_{n}, u_{n-1}\right)+\varphi\left(u_{n-1}\right)-\varphi\left(u_{n}\right)+\left\langle D x_{n}, u_{n-1}-u_{n}\right\rangle+\frac{1}{r_{n}}\left\langle u_{n-1}-u_{n}, u_{n}-x_{n}\right\rangle \geq 0
$$

and

$$
\begin{aligned}
& F\left(u_{n-1}, u_{n}\right)+\varphi\left(u_{n}\right)-\varphi\left(u_{n-1}\right)+\left\langle D x_{n-1}, u_{n}-u_{n-1}\right\rangle \\
& +\frac{1}{r_{n-1}}\left\langle u_{n}-u_{n-1}, u_{n-1}-x_{n-1}\right\rangle \geq 0 .
\end{aligned}
$$

Adding (3.8) and (3.9) and using the monotonicity of $F$, we have

$$
\left\langle D x_{n-1}-D x_{n}, u_{n}-u_{n-1}\right\rangle+\left\langle u_{n}-u_{n-1}, \frac{u_{n-1}-x_{n-1}}{r_{n-1}}-\frac{u_{n}-x_{n}}{r_{n}}\right\rangle \geq 0,
$$

which implies that

$$
\begin{aligned}
0 \leq & \left\langle u_{n}-u_{n-1}, r_{n}\left(D x_{n-1}-D x_{n}\right)+\frac{r_{n}}{r_{n-1}}\left(u_{n-1}-x_{n-1}\right)-\left(u_{n}-x_{n}\right)\right\rangle \\
= & \left\langle u_{n-1}-u_{n}, u_{n}-u_{n-1}+\left(1-\frac{r_{n}}{r_{n-1}}\right) u_{n-1}\right. \\
& \left.+\left(x_{n-1}-r_{n} D x_{n-1}\right)-\left(x_{n}-r_{n} D x_{n}\right)-x_{n-1}+\frac{r_{n}}{r_{n-1}} x_{n-1}\right\rangle \\
= & \left\langle u_{n-1}-u_{n},\left(1-\frac{r_{n}}{r_{n-1}}\right) u_{n-1}+\left(x_{n-1}-r_{n} D x_{n-1}\right)-\left(x_{n}-r_{n} D x_{n}\right)-x_{n-1}+\frac{r_{n}}{r_{n-1}} x_{n-1}\right\rangle \\
& -\left\|u_{n}-u_{n-1}\right\|^{2} \\
= & \left\langle u_{n-1}-u_{n},\left(1-\frac{r_{n}}{r_{n-1}}\right)\left(u_{n-1}-x_{n-1}\right)+\left(x_{n-1}-r_{n} D x_{n-1}\right)-\left(x_{n}-r_{n} D x_{n}\right)\right\rangle \\
& -\left\|u_{n}-u_{n-1}\right\|^{2} \\
\leq & \left\|u_{n}-u_{n-1}\right\|\left\{\left|1-\frac{r_{n}}{r_{n-1}}\right|\left\|u_{n-1}-x_{n-1}\right\|+\left\|\left(x_{n-1}-r_{n} D x_{n-1}\right)-\left(x_{n}-r_{n} D x_{n}\right)\right\|\right\} \\
& -\left\|u_{n}-u_{n-1}\right\|^{2} \\
= & \left\|u_{n}-u_{n-1}\right\|\left\{\left|1-\frac{r_{n}}{r_{n-1}}\right|\left\|u_{n-1}-x_{n-1}\right\|+\left\|x_{n-1}-x_{n}\right\|\right\}-\left\|u_{n}-u_{n-1}\right\|^{2},
\end{aligned}
$$


and then

$$
\left\|u_{n}-u_{n-1}\right\| \leq\left|1-\frac{r_{n}}{r_{n-1}}\right|\left\|u_{n-1}-x_{n-1}\right\|+\left\|x_{n-1}-x_{n}\right\| .
$$

Without loss of generality, let us assume that there exists a real number $\mu$ such that $r_{n}>$ $\mu>0$ for all positive integers $n$. Then we get

$$
\left\|u_{n-1}-u_{n}\right\| \leq\left\|x_{n-1}-x_{n}\right\|+\frac{1}{\mu}\left|r_{n-1}-r_{n}\right|\left\|u_{n-1}-x_{n-1}\right\| .
$$

It follows from (3.5) and (3.10) that

$$
\begin{aligned}
\| y_{n}- & y_{n-1} \| \\
\leq & \beta_{n}\left\|x_{n}-x_{n-1}\right\|+\left(1-\beta_{n}\right)\left\{\left\|x_{n}-x_{n-1}\right\|+\frac{1}{\mu}\left|r_{n}-r_{n-1}\right|\left\|u_{n-1}-x_{n-1}\right\|\right. \\
& \left.+M \cdot \sum_{m=1}^{r}\left|\eta_{n}^{m}-\eta_{n-1}^{m}\right|+\left|\delta_{n}-\delta_{n-1}\right|\left\|u_{n-1}-z_{n-1}\right\|\right\}+\left|\beta_{n}-\beta_{n-1}\right|\left(\left\|S x_{n-1}\right\|+\left\|v_{n-1}\right\|\right) \\
= & \left\|x_{n}-x_{n-1}\right\|+\left(1-\beta_{n}\right)\left\{\frac{1}{\mu}\left|r_{n}-r_{n-1}\right|\left\|u_{n-1}-x_{n-1}\right\|+M \cdot \sum_{m=1}^{r}\left|\eta_{n}^{m}-\eta_{n-1}^{m}\right|\right. \\
& \left.+\left|\delta_{n}-\delta_{n-1}\right|\left\|u_{n-1}-z_{n-1}\right\|\right\}+\left|\beta_{n}-\beta_{n-1}\right|\left(\left\|S x_{n-1}\right\|+\left\|v_{n-1}\right\|\right) \\
\leq & \left\|x_{n}-x_{n-1}\right\|+\frac{1}{\mu}\left|r_{n}-r_{n-1}\right|\left\|u_{n-1}-x_{n-1}\right\|+M \cdot \sum_{m=1}^{r}\left|\eta_{n}^{m}-\eta_{n-1}^{m}\right| \\
& +\left|\delta_{n}-\delta_{n-1}\right|\left\|u_{n-1}-z_{n-1}\right\|+\left|\beta_{n}-\beta_{n-1}\right|\left(\left\|S x_{n-1}\right\|+\left\|v_{n-1}\right\|\right) .
\end{aligned}
$$

Next, we estimate

$$
\begin{aligned}
&\left\|x_{n+1}-x_{n}\right\| \\
&=\left\|P_{C}\left[V_{n}\right]-P_{C}\left[V_{n-1}\right]\right\| \\
& \leq \| \alpha_{n} \rho\left(U\left(x_{n}\right)-U\left(x_{n-1}\right)\right)+\left(\alpha_{n}-\alpha_{n-1}\right) \rho U\left(x_{n-1}\right) \\
& \quad+\gamma_{n}\left(x_{n}-x_{n-1}\right)+\left(\gamma_{n}-\gamma_{n-1}\right) x_{n-1} \\
& \quad+\left(1-\gamma_{n}\right)\left[\left(I-\frac{\alpha_{n} \mu}{1-\gamma_{n}} F\right) T\left(y_{n}\right)-\left(I-\frac{\alpha_{n} \mu}{1-\gamma_{n}} F\right) T\left(y_{n-1}\right)\right] \\
& \quad+\left(\left(1-\gamma_{n}\right) I-\alpha_{n} \mu F\right) T\left(y_{n-1}\right)-\left(\left(1-\gamma_{n-1}\right) I-\alpha_{n-1} \mu F\right) T\left(y_{n-1}\right) \| \\
& \leq \alpha_{n} \rho \tau\left\|x_{n}-x_{n-1}\right\|+\gamma_{n}\left\|x_{n}-x_{n-1}\right\|+\left(1-\gamma_{n}\right)\left(1-\frac{\alpha_{n} v}{1-\gamma_{n}}\right)\left\|y_{n}-y_{n-1}\right\| \\
&+\left|\gamma_{n}-\gamma_{n-1}\right|\left(\left\|x_{n-1}\right\|+\left\|T\left(y_{n-1}\right)\right\|\right) \\
&+\left|\alpha_{n}-\alpha_{n-1}\right|\left(\rho\left\|U\left(x_{n-1}\right)\right\|+\left\|\mu F\left(T\left(y_{n-1}\right)\right)\right\|\right),
\end{aligned}
$$


which the second inequality follows from Lemma 2.5. From (3.11) and (3.12), we have

$$
\begin{aligned}
\| x_{n+1} & -x_{n} \| \\
\leq & \alpha_{n} \rho \tau\left\|x_{n}-x_{n-1}\right\|+\gamma_{n}\left\|x_{n}-x_{n-1}\right\|+\left(1-\gamma_{n}-\alpha_{n} v\right) \\
& \times\left\{\left\|x_{n}-x_{n-1}\right\|+\frac{1}{\mu}\left|r_{n}-r_{n-1}\right|\left\|u_{n-1}-x_{n-1}\right\|+M \cdot \sum_{m=1}^{r}\left|\eta_{n}^{m}-\eta_{n-1}^{m}\right|\right. \\
& \left.+\left|\delta_{n}-\delta_{n-1}\right|\left\|u_{n-1}-z_{n-1}\right\|+\left|\beta_{n}-\beta_{n-1}\right|\left(\left\|S x_{n-1}\right\|+\left\|v_{n-1}\right\|\right)\right\} \\
& +\left|\gamma_{n}-\gamma_{n-1}\right|\left(\left\|x_{n-1}\right\|+\left\|T\left(y_{n-1}\right)\right\|\right)+\left|\alpha_{n}-\alpha_{n-1}\right|\left(\rho\left\|U\left(x_{n-1}\right)\right\|+\left\|\mu F\left(T\left(y_{n-1}\right)\right)\right\|\right) \\
\leq & (1-(v-\rho \tau))\left\|x_{n}-x_{n-1}\right\|+\frac{1}{\mu}\left|r_{n}-r_{n-1}\right||| u_{n-1}-x_{n-1} \|+M \cdot \sum_{m=1}^{r}\left|\eta_{n}^{m}-\eta_{n-1}^{m}\right| \\
& +\left|\delta_{n}-\delta_{n-1}\right|\left\|u_{n-1}-z_{n-1}\right\|+\left|\beta_{n}-\beta_{n-1}\right|\left(\left\|S x_{n-1}\right\|+\left\|v_{n-1}\right\|\right) \\
& +\left|\gamma_{n}-\gamma_{n-1}\right|\left(\left\|x_{n-1}\right\|+\left\|T\left(y_{n-1}\right)\right\|\right) \\
& +\left|\alpha_{n}-\alpha_{n-1}\right|\left(\rho\left\|U\left(x_{n-1}\right)\right\|+\left\|\mu F\left(T\left(y_{n-1}\right)\right)\right\|\right) \\
\leq & (1-(v-\rho \tau))\left\|x_{n}-x_{n-1}\right\|+M \cdot \sum_{m=1}^{r}\left|\eta_{n}^{m}-\eta_{n-1}^{m}\right|+M_{1}\left(\frac{1}{\mu}\left|r_{n}-r_{n-1}\right|\right. \\
& \left.+\left|\delta_{n}-\delta_{n-1}\right|+\left|\beta_{n}-\beta_{n-1}\right|+\left|\gamma_{n}-\gamma_{n-1}\right|+\left|\alpha_{n}-\alpha_{n-1}\right|\right),
\end{aligned}
$$

where

$$
\begin{aligned}
M_{1}= & \max \left\{\sup _{n \geq 1}\left\|u_{n-1}-x_{n-1}\right\|, \sup _{n \geq 1}\left\|u_{n-1}-z_{n-1}\right\|, \sup _{n \geq 1}\left(\left\|S x_{n-1}\right\|+\left\|v_{n-1}\right\|\right),\right. \\
& \left.\sup _{n \geq 1}\left(\left\|x_{n-1}\right\|+\left\|T\left(y_{n-1}\right)\right\|\right),\left(\rho\left\|U\left(x_{n-1}\right)\right\|+\left\|\mu F\left(T\left(y_{n-1}\right)\right)\right\|\right)\right\} .
\end{aligned}
$$

It follows by condition (a)-(e) of Algorithm 3.1 and Lemma 2.6 that

$$
\lim _{n \rightarrow \infty}\left\|x_{n+1}-x_{n}\right\|=0 .
$$

Next, we show that $\lim _{n \rightarrow \infty}\left\|u_{n}-x_{n}\right\|=0$. Since $x^{*} \in \mathscr{F}=F(T) \cap \operatorname{VI}\left(C, B_{m}\right) \cap \operatorname{GMEP}(F$, $\varphi, D)$, by using (3.1) and (3.2), we obtain

$$
\begin{aligned}
\left\|x_{n+1}-x^{*}\right\|^{2}= & \left\langle P_{C}\left[V_{n}\right]-x^{*}, x_{n+1}-x^{*}\right\rangle \\
= & \left\langle P_{C}\left[V_{n}\right]-V_{n}, P_{C}\left[V_{n}\right]-x^{*}\right\rangle+\left\langle V_{n}-x^{*}, x_{n+1}-x^{*}\right\rangle \\
\leq & \left\langle\alpha_{n}\left(\rho U\left(x_{n}\right)-\mu F\left(x^{*}\right)\right)+\gamma_{n}\left(x_{n}-x^{*}\right)+\left(\left(1-\gamma_{n}\right) I-\alpha_{n} \mu F\right) T\left(y_{n}\right)\right. \\
& \left.-\left(\left(1-\gamma_{n}\right) I-\alpha_{n} \mu F\right) T\left(x^{*}\right), x_{n+1}-x^{*}\right\rangle \\
= & \left\langle\alpha_{n} \rho\left(U\left(x_{n}\right)-U\left(x^{*}\right)\right), x_{n+1}-x^{*}\right\rangle+\alpha_{n}\left\langle\rho U\left(x^{*}\right)-\mu F\left(x^{*}\right), x_{n+1}-x^{*}\right\rangle \\
& +\left\langle\gamma_{n}\left(x_{n}-x^{*}\right), x_{n+1}-x^{*}\right\rangle \\
& +\left(1-\gamma_{n}\right)\left\langle\left(I-\frac{\alpha_{n} \mu}{1-\gamma_{n}} F\right) T\left(y_{n}\right)-\left(I-\frac{\alpha_{n} \mu}{1-\gamma_{n}} F\right) T\left(x^{*}\right), x_{n+1}-x^{*}\right\rangle
\end{aligned}
$$




$$
\begin{aligned}
\leq & \left(\alpha_{n} \rho \tau+\gamma_{n}\right)\left\|x_{n}-x^{*}\right\|\left\|x_{n+1}-x^{*}\right\|+\alpha_{n}\left(\rho U\left(x^{*}\right)-\mu F\left(x^{*}\right), x_{n+1}-x^{*}\right\rangle \\
& +\left(1-\gamma_{n}-\alpha_{n} v\right)\left\|y_{n}-x^{*}\right\|\left\|x_{n+1}-x^{*}\right\| \\
\leq & \frac{\gamma_{n}+\alpha_{n} \rho \tau}{2}\left(\left\|x_{n}-x^{*}\right\|^{2}+\left\|x_{n+1}-x^{*}\right\|^{2}\right) \\
& +\alpha_{n}\left\langle\rho U\left(x^{*}\right)-\mu F\left(x^{*}\right), x_{n+1}-x^{*}\right\rangle \\
& +\frac{1-\gamma_{n}-\alpha_{n} v}{2}\left(\left\|y_{n}-x^{*}\right\|^{2}+\left\|x_{n+1}-x^{*}\right\|^{2}\right) \\
\leq & \frac{1-\alpha_{n}(v-\rho \tau)}{2}\left\|x_{n+1}-x^{*}\right\|^{2}+\frac{\gamma_{n}+\alpha_{n} \rho \tau}{2}\left\|x_{n}-x^{*}\right\|^{2} \\
& +\alpha_{n}\left(\rho U\left(x^{*}\right)-\mu F\left(x^{*}\right), x_{n+1}-x^{*}\right\rangle \\
& +\frac{1-\gamma_{n}-\alpha_{n} v}{2}\left(\beta_{n}\left\|S x_{n}-x^{*}\right\|^{2}+\left(1-\beta_{n}\right)\left\|v_{n}-x^{*}\right\|^{2}\right) \\
\leq & \frac{1-\alpha_{n}(v-\rho \tau)}{2}\left\|x_{n+1}-x^{*}\right\|^{2}+\frac{\gamma_{n}+\alpha_{n} \rho \tau}{2}\left\|x_{n}-x^{*}\right\|^{2} \\
& +\alpha_{n}\left\langle\rho U\left(x^{*}\right)-\mu F\left(x^{*}\right), x_{n+1}-x^{*}\right\rangle+\frac{\left(1-\gamma_{n}-\alpha_{n} v\right) \beta_{n}}{2}\left\|S x_{n}-x^{*}\right\|^{2} \\
& +\frac{\left(1-\gamma_{n}-\alpha_{n} v\right)\left(1-\beta_{n}\right)}{2} \\
& \times\left\{\left\|x_{n}-x^{*}\right\|^{2}-r_{n}\left(2 \theta-r_{n}\right)\left\|D x_{n}-D x^{*}\right\|^{2}\right\},
\end{aligned}
$$

which implies that

$$
\begin{aligned}
\left\|x_{n+1}-x^{*}\right\|^{2} \leq & \frac{\gamma_{n}+\alpha_{n} \rho \tau}{1+\alpha_{n}(v-\rho \tau)}\left\|x_{n}-x^{*}\right\|^{2} \\
& +\frac{2 \alpha_{n}}{1+\alpha_{n}(v-\rho \tau)}\left\langle\rho U\left(x^{*}\right)-\mu F\left(x^{*}\right), x_{n+1}-x^{*}\right\rangle \\
& +\frac{\left(1-\gamma_{n}-\alpha_{n} v\right) \beta_{n}}{1+\alpha_{n}(v-\rho \tau)}\left\|S x_{n}-x^{*}\right\|^{2} \\
& +\frac{\left(1-\gamma_{n}-\alpha_{n} v\right)\left(1-\beta_{n}\right)}{1+\alpha_{n}(v-\rho \tau)}\left\{\left\|x_{n}-x^{*}\right\|^{2}-r_{n}\left(2 \theta-r_{n}\right)\left\|D x_{n}-D x^{*}\right\|^{2}\right\} \\
\leq & \frac{\gamma_{n}+\alpha_{n} \rho \tau}{1+\alpha_{n}(v-\rho \tau)}\left\|x_{n}-x^{*}\right\|^{2} \\
& +\frac{2 \alpha_{n}}{1+\alpha_{n}(v-\rho \tau)}\left\langle\rho U\left(x^{*}\right)-\mu F\left(x^{*}\right), x_{n+1}-x^{*}\right\rangle \\
& +\left\|x_{n}-x^{*}\right\|^{2}+\frac{\left(1-\gamma_{n}-\alpha_{n} v\right) \beta_{n}}{1+\alpha_{n}(v-\rho \tau)}\left\|S x_{n}-x^{*}\right\|^{2} \\
& -\frac{\left(1-\gamma_{n}-\alpha_{n} v\right)\left(1-\beta_{n}\right)}{1+\alpha_{n}(v-\rho \tau)}\left\{r_{n}\left(2 \theta-r_{n}\right)\left\|D x_{n}-D x^{*}\right\|^{2}\right\} .
\end{aligned}
$$

Then from the above inequality, we get

$$
\begin{aligned}
& \frac{\left(1-\gamma_{n}-\alpha_{n} v\right)\left(1-\beta_{n}\right)}{1+\alpha_{n}(v-\rho \tau)}\left\{r_{n}\left(2 \theta-r_{n}\right)\left\|D x_{n}-D x^{*}\right\|^{2}\right\} \\
& \leq \frac{\gamma_{n}+\alpha_{n} \rho \tau}{1+\alpha_{n}(v-\rho \tau)}\left\|x_{n}-x^{*}\right\|^{2}+\frac{2 \alpha_{n}}{1+\alpha_{n}(v-\rho \tau)}\left\langle\rho U\left(x^{*}\right)-\mu F\left(x^{*}\right), x_{n+1}-x^{*}\right\rangle \\
& \quad+\beta_{n}\left\|S x_{n}-x^{*}\right\|^{2}+\left\|x_{n}-x^{*}\right\|^{2}-\left\|x_{n+1}-x^{*}\right\|^{2}
\end{aligned}
$$




$$
\begin{aligned}
\leq & \frac{\gamma_{n}+\alpha_{n} \rho \tau}{1+\alpha_{n}(v-\rho \tau)}\left\|x_{n}-x^{*}\right\|^{2}+\frac{2 \alpha_{n}}{1+\alpha_{n}(v-\rho \tau)}\left\langle\rho U\left(x^{*}\right)-\mu F\left(x^{*}\right), x_{n+1}-x^{*}\right\rangle \\
& +\beta_{n}\left\|S x_{n}-x^{*}\right\|^{2}+\left(\left\|x_{n}-x^{*}\right\|+\left\|x_{n+1}-x^{*}\right\|\right)\left\|x_{n+1}-x_{n}\right\| .
\end{aligned}
$$

Since $\left\{r_{n}\right\} \subset(0,2 \theta), \lim _{n \rightarrow \infty}\left\|x_{n+1}-x_{n}\right\|=0, \gamma_{n} \rightarrow 0, \alpha_{n} \rightarrow 0$, and $\beta_{n} \rightarrow 0$, we obtain $\lim _{n \rightarrow \infty}\left\|D x_{n}-D x^{*}\right\|=0$.

Since $T_{r_{n}}$ is firmly nonexpansive, we have

$$
\begin{aligned}
\left\|u_{n}-x^{*}\right\|^{2}= & \left\|T_{r_{n}}\left(x_{n}-r_{n} D x_{n}\right)-T_{r_{n}}\left(x^{*}-r_{n} D x^{*}\right)\right\|^{2} \\
\leq & \left\langle u_{n}-x^{*},\left(x_{n}-r_{n} D x_{n}\right)-\left(x^{*}-r_{n} D x^{*}\right)\right\rangle \\
= & \frac{1}{2}\left\{\left\|u_{n}-x^{*}\right\|^{2}+\left\|\left(x_{n}-r_{n} D x_{n}\right)-\left(x^{*}-r_{n} D x^{*}\right)\right\|^{2}\right. \\
& \left.-\left\|u_{n}-x^{*}-\left[\left(x_{n}-r_{n} D x_{n}\right)-\left(x^{*}-r_{n} D x^{*}\right)\right]\right\|^{2}\right\} .
\end{aligned}
$$

Hence, we get

$$
\begin{aligned}
\left\|u_{n}-x^{*}\right\|^{2} & \leq\left\|\left(x_{n}-r_{n} D x_{n}\right)-\left(x^{*}-r_{n} D x^{*}\right)\right\|^{2}-\left\|u_{n}-x_{n}+r_{n}\left(D x_{n}-D x^{*}\right)\right\|^{2} \\
& \leq\left\|x_{n}-x^{*}\right\|^{2}-\left\|u_{n}-x_{n}+r_{n}\left(D x_{n}-D x^{*}\right)\right\|^{2} \\
& \leq\left\|x_{n}-x^{*}\right\|^{2}-\left\|u_{n}-x_{n}\right\|^{2}+2 r_{n}\left\|u_{n}-x_{n}\right\|\left\|D x_{n}-D x^{*}\right\| .
\end{aligned}
$$

From (3.14), (3.2), and the above inequality, we have

$$
\begin{aligned}
\left\|x_{n+1}-x^{*}\right\|^{2} \leq & \frac{1-\alpha_{n}(v-\rho \tau)}{2}\left\|x_{n+1}-x^{*}\right\|^{2}+\frac{\gamma_{n}+\alpha_{n} \rho \tau}{2}\left\|x_{n}-x^{*}\right\|^{2} \\
& +\alpha_{n}\left\{\rho U\left(x^{*}\right)-\mu F\left(x^{*}\right), x_{n+1}-x^{*}\right\rangle \\
& +\frac{1-\gamma_{n}-\alpha_{n} v}{2}\left(\beta_{n}\left\|S x_{n}-x^{*}\right\|^{2}+\left(1-\beta_{n}\right)\left\|v_{n}-x^{*}\right\|^{2}\right) \\
\leq & \frac{1-\alpha_{n}(v-\rho \tau)}{2}\left\|x_{n+1}-x^{*}\right\|^{2}+\frac{\gamma_{n}+\alpha_{n} \rho \tau}{2}\left\|x_{n}-x^{*}\right\|^{2} \\
& +\alpha_{n}\left\{\rho U\left(x^{*}\right)-\mu F\left(x^{*}\right), x_{n+1}-x^{*}\right\rangle \\
& +\frac{1-\gamma_{n}-\alpha_{n} v}{2}\left(\beta_{n}\left\|S x_{n}-x^{*}\right\|^{2}+\left(1-\beta_{n}\right)\left\|u_{n}-x^{*}\right\|^{2}\right) \\
\leq & \frac{1-\alpha_{n}(v-\rho \tau)}{2}\left\|x_{n+1}-x^{*}\right\|^{2}+\frac{\gamma_{n}+\alpha_{n} \rho \tau}{2}\left\|x_{n}-x^{*}\right\|^{2} \\
& +\alpha_{n}\left\{\rho U\left(x^{*}\right)-\mu F\left(x^{*}\right), x_{n+1}-x^{*}\right\rangle \\
& +\frac{1-\gamma_{n}-\alpha_{n} v}{2}\left\{\beta_{n}\left\|S x_{n}-x^{*}\right\|^{2}+\left(1-\beta_{n}\right)\left(\left\|x_{n}-x^{*}\right\|^{2}-\left\|u_{n}-x_{n}\right\|^{2}\right.\right. \\
& \left.\left.+2 r_{n}\left\|u_{n}-x_{n}\right\|\left\|D x_{n}-D x^{*}\right\|\right)\right\},
\end{aligned}
$$

which implies

$$
\begin{aligned}
\left\|x_{n+1}-x^{*}\right\|^{2} \leq & \frac{\gamma_{n}+\alpha_{n} \rho \tau}{1+\alpha_{n}(v-\rho \tau)}\left\|x_{n}-x^{*}\right\|^{2} \\
& +\frac{2 \alpha_{n}}{1+\alpha_{n}(v-\rho \tau)}\left\langle\rho U\left(x^{*}\right)-\mu F\left(x^{*}\right), x_{n+1}-x^{*}\right\rangle
\end{aligned}
$$




$$
\begin{aligned}
& +\frac{\left(1-\gamma_{n}-\alpha_{n} v\right) \beta_{n}}{1+\alpha_{n}(v-\rho \tau)}\left\|S x_{n}-x^{*}\right\|^{2} \\
+ & \frac{\left(1-\gamma_{n}-\alpha_{n} v\right)\left(1-\beta_{n}\right)}{1+\alpha_{n}(v-\rho \tau)}\left\{\left\|x_{n}-x^{*}\right\|^{2}-\left\|u_{n}-x_{n}\right\|^{2}\right. \\
& \left.+2 r_{n}\left\|u_{n}-x_{n}\right\|\left\|D x_{n}-D x^{*}\right\|\right\} \\
\leq & \frac{\gamma_{n}+\alpha_{n} \rho \tau}{1+\alpha_{n}(v-\rho \tau)}\left\|x_{n}-x^{*}\right\|^{2} \\
& +\frac{2 \alpha_{n}}{1+\alpha_{n}(v-\rho \tau)}\left\{\rho U\left(x^{*}\right)-\mu F\left(x^{*}\right), x_{n+1}-x^{*}\right\rangle \\
& +\frac{\left(1-\gamma_{n}-\alpha_{n} v\right) \beta_{n}}{1+\alpha_{n}(v-\rho \tau)}\left\|S x_{n}-x^{*}\right\|^{2} \\
& +\left\|x_{n}-x^{*}\right\|^{2}+\frac{\left(1-\gamma_{n}-\alpha_{n} v\right)\left(1-\beta_{n}\right)}{1+\alpha_{n}(v-\rho \tau)} \\
& \times\left\{-\left\|u_{n}-x_{n}\right\|^{2}+2 r_{n}\left\|u_{n}-x_{n}\right\|\left\|D x_{n}-D x^{*}\right\|\right\} .
\end{aligned}
$$

Hence

$$
\begin{aligned}
& \frac{\left(1-\gamma_{n}-\alpha_{n} v\right)\left(1-\beta_{n}\right)}{1+\alpha_{n}(v-\rho \tau)}\left\|u_{n}-x_{n}\right\|^{2} \\
& \leq \frac{\gamma_{n}+\alpha_{n} \rho \tau}{1+\alpha_{n}(v-\rho \tau)}\left\|x_{n}-x^{*}\right\|^{2}+\frac{2 \alpha_{n}}{1+\alpha_{n}(v-\rho \tau)}\left\langle\rho U\left(x^{*}\right)-\mu F\left(x^{*}\right), x_{n+1}-x^{*}\right\rangle \\
& \quad+\frac{\left(1-\gamma_{n}-\alpha_{n} v\right) \beta_{n}}{1+\alpha_{n}(v-\rho \tau)}\left\|S x_{n}-x^{*}\right\|^{2} \\
& \quad+\frac{2\left(1-\gamma_{n}-\alpha_{n} v\right)\left(1-\beta_{n}\right) r_{n}}{1+\alpha_{n}(v-\rho \tau)}\left\|u_{n}-x_{n}\right\|\left\|D x_{n}-D x^{*}\right\|+\left\|x_{n}-x^{*}\right\|^{2}-\left\|x_{n+1}-x^{*}\right\|^{2} \\
& \leq \\
& \quad \frac{\gamma_{n}+\alpha_{n} \rho \tau}{1+\alpha_{n}(v-\rho \tau)}\left\|x_{n}-x^{*}\right\|^{2}+\frac{2 \alpha_{n}}{1+\alpha_{n}(v-\rho \tau)}\left\langle\rho U\left(x^{*}\right)-\mu F\left(x^{*}\right), x_{n+1}-x^{*}\right\rangle \\
& \quad+\frac{\left(1-\gamma_{n}-\alpha_{n} v\right) \beta_{n}}{1+\alpha_{n}(v-\rho \tau)}\left\|S x_{n}-x^{*}\right\|^{2} \\
& \quad+\frac{2\left(1-\gamma_{n}-\alpha_{n} v\right)\left(1-\beta_{n}\right) r_{n}}{1+\alpha_{n}(v-\rho \tau)}\left\|u_{n}-x_{n}\right\|\left\|D x_{n}-D x^{*}\right\| \\
& \quad+\left(\left\|x_{n}-x^{*}\right\|+\left\|x_{n+1}-x^{*}\right\|\right)\left(\left\|x_{n+1}-x_{n}\right\|\right) .
\end{aligned}
$$

Since $\lim _{n \rightarrow \infty}\left\|x_{n+1}-x_{n}\right\|=0, \gamma_{n} \rightarrow 0, \alpha_{n} \rightarrow 0, \beta_{n} \rightarrow 0$, and $\lim _{n \rightarrow \infty}\left\|D x_{n}-D x^{*}\right\|=0$, we obtain

$$
\lim _{n \rightarrow \infty}\left\|u_{n}-x_{n}\right\|=0
$$

\section{Consider}

$$
\begin{aligned}
\left\|w_{n}^{m}-x^{*}\right\|^{2} & =\left\|P_{C}\left(I-\mu_{m} B_{m}\right) u_{n}-P_{C}\left(I-\mu_{m} B_{m}\right) x^{*}\right\|^{2} \\
& \leq\left\|\left(u_{n}-x^{*}\right)-\mu_{m}\left(B_{m} u_{n}-B_{m} x^{*}\right)\right\|^{2} \\
& =\left\|u_{n}-x^{*}\right\|^{2}+\mu_{m}^{2}\left\|B_{m} u_{n}-B_{m} x^{*}\right\|^{2}-2 \mu_{m}\left\langle u_{n}-x^{*}, B_{m} u_{n}-B_{m} x^{*}\right\rangle \\
& \leq\left\|u_{n}-x^{*}\right\|^{2}+\mu_{m}^{2}\left\|B_{m} u_{n}-B_{m} x^{*}\right\|^{2}-2 \mu_{m} l_{m}\left\|B_{m} u_{n}-B_{m} x^{*}\right\|^{2} \\
& \leq\left\|u_{n}-x^{*}\right\|^{2}-\mu_{m}\left(2 l_{m}-\mu_{m}\right)\left\|B_{m} u_{n}-B_{m} x^{*}\right\|^{2}, \quad 1 \leq \forall m \leq r .
\end{aligned}
$$


It follows that

$$
\begin{aligned}
\left\|z_{n}-x^{*}\right\|^{2} & =\left\|\sum_{m=1}^{r}\left(\eta_{n}^{m} w_{n}^{m}\right)-x^{*}\right\|^{2} \leq \sum_{m=1}^{r} \eta_{n}^{m}\left\|w_{n}^{m}-x^{*}\right\|^{2} \\
& \leq\left\|u_{n}-x^{*}\right\|^{2}-\sum_{m=1}^{r} \eta_{n}^{m} \mu_{m}\left(2 l_{m}-\mu_{m}\right)\left\|B_{m} u_{n}-B_{m} x^{*}\right\|^{2} .
\end{aligned}
$$

Then we have

$$
\begin{aligned}
\left\|v_{n}-x^{*}\right\|^{2} & =\left\|\delta_{n}\left(u_{n}-x^{*}\right)+\left(1-\delta_{n}\right)\left(z_{n}-x^{*}\right)\right\|^{2} \\
& \leq \delta_{n}\left\|u_{n}-x^{*}\right\|^{2}+\left(1-\delta_{n}\right)\left\|z_{n}-x^{*}\right\|^{2} \\
& =\left\|u_{n}-x^{*}\right\|^{2}-\left(1-\delta_{n}\right) \sum_{m=1}^{r} \eta_{n}^{m} \mu_{m}\left(2 l_{m}-\mu_{m}\right)\left\|B_{m} u_{n}-B_{m} x^{*}\right\|^{2} \\
& \leq\left\|x_{n}-x^{*}\right\|^{2}-\left(1-\delta_{n}\right) \sum_{m=1}^{r} \eta_{n}^{m} \mu_{m}\left(2 l_{m}-\mu_{m}\right)\left\|B_{m} u_{n}-B_{m} x^{*}\right\|^{2} .
\end{aligned}
$$

From (3.14) and the above inequality, we have

$$
\begin{aligned}
\left\|x_{n+1}-x^{*}\right\|^{2} \leq & \frac{1-\alpha_{n}(v-\rho \tau)}{2}\left\|x_{n+1}-x^{*}\right\|^{2}+\frac{\gamma_{n}+\alpha_{n} \rho \tau}{2}\left\|x_{n}-x^{*}\right\|^{2} \\
& +\alpha_{n}\left(\rho U\left(x^{*}\right)-\mu F\left(x^{*}\right), x_{n+1}-x^{*}\right\rangle \\
& +\frac{1-\gamma_{n}-\alpha_{n} v}{2}\left(\beta_{n}\left\|S x_{n}-x^{*}\right\|^{2}+\left(1-\beta_{n}\right)\left\|v_{n}-x^{*}\right\|^{2}\right) \\
\leq & \frac{1-\alpha_{n}(v-\rho \tau)}{2}\left\|x_{n+1}-x^{*}\right\|^{2}+\frac{\gamma_{n}+\alpha_{n} \rho \tau}{2}\left\|x_{n}-x^{*}\right\|^{2} \\
& +\alpha_{n}\left(\rho U\left(x^{*}\right)-\mu F\left(x^{*}\right), x_{n+1}-x^{*}\right\rangle \\
& +\frac{\left(1-\gamma_{n}-\alpha_{n} v\right)}{2}\left\{\beta_{n}\left\|S x_{n}-x^{*}\right\|^{2}+\left(1-\beta_{n}\right)\left(\left\|x_{n}-x^{*}\right\|^{2}\right.\right. \\
& \left.\left.-\left(1-\delta_{n}\right) \sum_{m=1}^{r} \eta_{n}^{m} \mu_{m}\left(2 l_{m}-\mu_{m}\right)\left\|B_{m} u_{n}-B_{m} x^{*}\right\|^{2}\right)\right\}
\end{aligned}
$$

which implies

$$
\begin{aligned}
\left\|x_{n+1}-x^{*}\right\|^{2} \leq & \frac{\gamma_{n}+\alpha_{n} \rho \tau}{1+\alpha_{n}(v-\rho \tau)}\left\|x_{n}-x^{*}\right\|^{2} \\
& +\frac{2 \alpha_{n}}{1+\alpha_{n}(v-\rho \tau)}\left\langle\rho U\left(x^{*}\right)-\mu F\left(x^{*}\right), x_{n+1}-x^{*}\right\rangle \\
& +\frac{\left(1-\gamma_{n}-\alpha_{n} v\right) \beta_{n}}{1+\alpha_{n}(v-\rho \tau)}\left\|S x_{n}-x^{*}\right\|^{2}+\left\|x_{n}-x^{*}\right\|^{2} \\
& -\frac{\left(1-\gamma_{n}-\alpha_{n} v\right)\left(1-\beta_{n}\right)\left(1-\delta_{n}\right)}{1+\alpha_{n}(v-\rho \tau)} \sum_{m=1}^{r} \eta_{n}^{m} \mu_{m}\left(2 l_{m}-\mu_{m}\right)\left\|B_{m} u_{n}-B_{m} x^{*}\right\|^{2} .
\end{aligned}
$$


Then from the above inequality, we have

$$
\begin{aligned}
& \frac{\left(1-\gamma_{n}-\alpha_{n} v\right)\left(1-\beta_{n}\right)\left(1-\delta_{n}\right)}{1+\alpha_{n}(v-\rho \tau)} \sum_{m=1}^{r} \eta_{n}^{m} \mu_{m}\left(2 l_{m}-\mu_{m}\right)\left\|B_{m} u_{n}-B_{m} x^{*}\right\|^{2} \\
& \leq \frac{\gamma_{n}+\alpha_{n} \rho \tau}{1+\alpha_{n}(v-\rho \tau)}\left\|x_{n}-x^{*}\right\|^{2}+\frac{2 \alpha_{n}}{1+\alpha_{n}(v-\rho \tau)}\left\langle\rho U\left(x^{*}\right)-\mu F\left(x^{*}\right), x_{n+1}-x^{*}\right\rangle \\
& \quad+\beta_{n}\left\|S x_{n}-x^{*}\right\|^{2}+\left\|x_{n}-x^{*}\right\|^{2}-\left\|x_{n+1}-x^{*}\right\|^{2} \\
& \leq \frac{\gamma_{n}+\alpha_{n} \rho \tau}{1+\alpha_{n}(v-\rho \tau)}\left\|x_{n}-x^{*}\right\|+\frac{2 \alpha_{n}}{1+\alpha_{n}(v-\rho \tau)}\left\langle\rho U\left(x^{*}\right)-\mu F\left(x^{*}\right), x_{n+1}-x^{*}\right\rangle \\
& \quad+\beta_{n}\left\|S x_{n}-x^{*}\right\|^{2}+\left(\left\|x_{n}-x^{*}\right\|+\left\|x_{n+1}-x^{*}\right\|\right)\left(\left\|x_{n+1}-x_{n}\right\|\right) .
\end{aligned}
$$

Since $\mu_{n} \in\left(0,2 l_{m}\right), \lim _{n \rightarrow \infty}\left\|x_{n+1}-x_{n}\right\|=0, \gamma_{n} \rightarrow 0, \alpha_{n} \rightarrow 0$, and $\beta_{n} \rightarrow 0$, we obtain

$$
\lim _{n \rightarrow \infty}\left\|B_{m} u_{n}-B_{m} x^{*}\right\|=0, \quad 1 \leq \forall m \leq r
$$

On the other hand, we have

$$
\begin{aligned}
&\left\|w_{n}^{m}-x^{*}\right\|^{2} \\
&=\left\|P_{C}\left(I-\mu_{m} B_{m}\right) u_{n}-P_{C}\left(I-\mu_{m} B_{m}\right) x^{*}\right\|^{2} \\
& \leq\left\langle\left(I-\mu_{m} B_{m}\right) u_{n}-\left(I-\mu_{m} B_{m}\right) x^{*}, w_{n}^{m}-x^{*}\right\rangle \\
&= \frac{1}{2}\left\{\left\|\left(I-\mu_{m} B_{m}\right) u_{n}-\left(I-\mu_{m} B_{m}\right) x^{*}\right\|^{2}+\left\|w_{n}^{m}-x^{*}\right\|^{2}\right. \\
&\left.-\left\|\left(I-\mu_{m} B_{m}\right) u_{n}-\left(I-\mu_{m} B_{m}\right) x^{*}-\left(w_{n}^{m}-x^{*}\right)\right\|^{2}\right\} \\
& \leq \frac{1}{2}\left\{\left\|u_{n}-x^{*}\right\|^{2}+\left\|w_{n}^{m}-x^{*}\right\|^{2}-\left\|u_{n}-w_{n}^{m}-\mu_{m}\left(B_{m} u_{n}-B_{m} x^{*}\right)\right\|^{2}\right\} \\
&= \frac{1}{2}\left\{\left\|u_{n}-x^{*}\right\|^{2}+\left\|w_{n}^{m}-x^{*}\right\|^{2}-\left\|u_{n}-w_{n}^{m}\right\|^{2}\right. \\
&\left.+2 \mu_{m}\left\langle B_{m} u_{n}-B_{m} x^{*}, u_{n}-w_{n}^{m}\right\rangle-\mu_{m}^{2}\left\|B_{m} u_{n}-B_{m} x^{*}\right\|^{2}\right\}, \quad 1 \leq \forall m \leq r .
\end{aligned}
$$

It follows that

$$
\left\|w_{n}^{m}-x^{*}\right\|^{2} \leq\left\|u_{n}-x^{*}\right\|^{2}-\left\|u_{n}-w_{n}^{m}\right\|^{2}+Q^{m}\left\|B_{m} u_{n}-B_{m} x^{*}\right\|, \quad 1 \leq \forall m \leq r,
$$

where $Q^{m}$ is an approximate constant such that

$$
Q^{m}=\max \left\{2 \mu_{m}\left\|u_{n}-w_{n}^{m}\right\|: \forall n \geq 1\right\}, \quad 1 \leq \forall m \leq r .
$$

On the other hand, we have

$$
\left\|z_{n}-u_{n}\right\|^{2} \leq \sum_{m=1}^{r}\left(\eta_{n}^{m}\left\|w_{n}^{m}-u_{n}\right\|^{2}\right)
$$


which combined with (3.16) gives

$$
\begin{aligned}
\left\|z_{n}-x^{*}\right\|^{2} & \leq \sum_{m=1}^{r}\left(\eta_{n}^{m}\left\|w_{n}^{m}-x^{*}\right\|^{2}\right) \\
& \leq\left\|u_{n}-x^{*}\right\|^{2}-\left\|z_{n}-u_{n}\right\|^{2}+\sum_{m=1}^{r}\left(Q^{m}\left\|B_{m} u_{n}-B_{m} x^{*}\right\|\right) .
\end{aligned}
$$

Hence we have

$$
\begin{aligned}
\left\|v_{n}-x^{*}\right\|^{2} & \leq \delta_{n}\left\|u_{n}-x^{*}\right\|^{2}+\left(1-\delta_{n}\right)\left\|z_{n}-x^{*}\right\|^{2} \\
& \leq\left\|u_{n}-x^{*}\right\|^{2}-\left\|z_{n}-u_{n}\right\|^{2}+\sum_{m=1}^{r}\left(Q^{m}\left\|B_{m} u_{n}-B_{m} x^{*}\right\|\right) \\
& \leq\left\|x_{n}-x^{*}\right\|^{2}-\left\|z_{n}-u_{n}\right\|^{2}+\sum_{m=1}^{r}\left(Q^{m}\left\|B_{m} u_{n}-B_{m} x^{*}\right\|\right) .
\end{aligned}
$$

In view of (3.14) and the above inequality, we have

$$
\begin{aligned}
\left\|x_{n+1}-x^{*}\right\|^{2} & \\
\leq & \frac{1-\alpha_{n}(v-\rho \tau)}{2}\left\|x_{n+1}-x^{*}\right\|^{2}+\frac{\gamma_{n}+\alpha_{n} \rho \tau}{2}\left\|x_{n}-x^{*}\right\|^{2} \\
& +\alpha_{n}\left\langle\rho U\left(x^{*}\right)-\mu F\left(x^{*}\right), x_{n+1}-x^{*}\right\rangle \\
& +\frac{1-\gamma_{n}-\alpha_{n} v}{2}\left(\beta_{n}\left\|S x_{n}-x^{*}\right\|^{2}+\left(1-\beta_{n}\right)\left\|v_{n}-x^{*}\right\|^{2}\right) \\
\leq & \frac{1-\alpha_{n}(v-\rho \tau)}{2}\left\|x_{n+1}-x^{*}\right\|^{2}+\frac{\gamma_{n}+\alpha_{n} \rho \tau}{2}\left\|x_{n}-x^{*}\right\|^{2} \\
& +\alpha_{n}\left\langle\rho U\left(x^{*}\right)-\mu F\left(x^{*}\right), x_{n+1}-x^{*}\right\rangle+\frac{1-\gamma_{n}-\alpha_{n} v}{2}\left\{\beta_{n}\left\|S x_{n}-x^{*}\right\|^{2}\right. \\
& \left.+\left(1-\beta_{n}\right)\left(\left\|x_{n}-x^{*}\right\|^{2}-\left\|z_{n}-u_{n}\right\|^{2}+\sum_{m=1}^{r}\left(Q^{m}\left\|B_{m} u_{n}-B_{m} x^{*}\right\|\right)\right)\right\},
\end{aligned}
$$

which implies that

$$
\begin{aligned}
\left\|x_{n+1}-x^{*}\right\|^{2} & \\
\leq & \frac{\gamma_{n}+\alpha_{n} \rho \tau}{1+\alpha_{n}(v-\rho \tau)}\left\|x_{n}-x^{*}\right\|^{2} \\
& +\frac{2 \alpha_{n}}{1+\alpha_{n}(v-\rho \tau)}\left\{\rho U\left(x^{*}\right)-\mu F\left(x^{*}\right), x_{n+1}-x^{*}\right\rangle \\
& +\frac{\left(1-\gamma_{n}-\alpha_{n} v\right) \beta_{n}}{1+\alpha_{n}(v-\rho \tau)}\left\|S x_{n}-x^{*}\right\|^{2} \\
& +\frac{\left(1-\gamma_{n}-\alpha_{n} v\right)\left(1-\beta_{n}\right)}{1+\alpha_{n}(v-\rho \tau)}\left\{\left\|x_{n}-x^{*}\right\|^{2}-\left\|z_{n}-u_{n}\right\|^{2}+\sum_{m=1}^{r}\left(Q^{m}\left\|B_{m} u_{n}-B_{m} x^{*}\right\|\right)\right\} .
\end{aligned}
$$


Hence

$$
\begin{aligned}
& \frac{\left(1-\gamma_{n}-\alpha_{n} v\right)\left(1-\beta_{n}\right)}{1+\alpha_{n}(v-\rho \tau)}\left\|z_{n}-u_{n}\right\|^{2} \\
& \leq \frac{\gamma_{n}+\alpha_{n} \rho \tau}{1+\alpha_{n}(v-\rho \tau)}\left\|x_{n}-x^{*}\right\|^{2}+\frac{2 \alpha_{n}}{1+\alpha_{n}(v-\rho \tau)}\left\langle\rho U\left(x^{*}\right)-\mu F\left(x^{*}\right), x_{n+1}-x^{*}\right\rangle \\
& \quad+\frac{\left(1-\gamma_{n}-\alpha_{n} v\right) \beta_{n}}{1+\alpha_{n}(v-\rho \tau)}\left\|S x_{n}-x^{*}\right\|^{2}+\left\|x_{n}-x^{*}\right\|^{2}-\left\|x_{n+1}-x^{*}\right\|^{2} \\
& \quad+\sum_{m=1}^{r}\left(Q^{m}\left\|B_{m} u_{n}-B_{m} x^{*}\right\|\right) \\
& =\frac{\gamma_{n}+\alpha_{n} \rho \tau}{1+\alpha_{n}(v-\rho \tau)}\left\|x_{n}-x^{*}\right\|^{2}+\frac{2 \alpha_{n}}{1+\alpha_{n}(v-\rho \tau)}\left\langle\rho U\left(x^{*}\right)-\mu F\left(x^{*}\right), x_{n+1}-x^{*}\right\rangle \\
& \quad+\frac{\left(1-\gamma_{n}-\alpha_{n} v\right) \beta_{n}}{1+\alpha_{n}(v-\rho \tau)}\left\|S x_{n}-x^{*}\right\|^{2}+\left(\left\|x_{n}-x^{*}\right\|+\left\|x_{n+1}-x^{*}\right\|\right)\left(\left\|x_{n+1}-x_{n}\right\|\right) \\
& \quad+\sum_{m=1}^{r}\left(Q^{m}\left\|B_{m} u_{n}-B_{m} x^{*}\right\|\right) .
\end{aligned}
$$

Since $\lim _{n \rightarrow \infty}\left\|x_{n+1}-x_{n}\right\|=0, \gamma_{n} \rightarrow 0, \alpha_{n} \rightarrow 0, \beta_{n} \rightarrow 0$, and $\lim _{n \rightarrow \infty}\left\|B_{m} u_{n}-B_{m} x^{*}\right\|=0$, we get

$$
\lim _{n \rightarrow \infty}\left\|z_{n}-u_{n}\right\|=0
$$

It follows from (3.15) and (3.18) that

$$
\lim _{n \rightarrow \infty}\left\|z_{n}-x_{n}\right\|=0
$$

From Algorithm 3.1, we have

$$
\left\|v_{n}-x_{n}\right\| \leq \delta_{n}\left\|u_{n}-x_{n}\right\|+\left(1-\delta_{n}\right)\left\|z_{n}-x_{n}\right\|,
$$

which implies

$$
\begin{aligned}
\lim _{n \rightarrow \infty}\left\|x_{n}-v_{n}\right\| & =0, \\
\left\|x_{n}-T\left(y_{n}\right)\right\| & \leq\left\|x_{n}-x_{n+1}\right\|+\left\|x_{n+1}-T\left(y_{n}\right)\right\| \\
& =\left\|x_{n}-x_{n+1}\right\|+\left\|P_{C}\left[V_{n}\right]-P_{C}\left[T\left(y_{n}\right)\right]\right\| \\
& \leq\left\|x_{n}-x_{n+1}\right\|+\left\|\alpha_{n}\left(\rho U\left(x_{n}\right)-\mu F\left(T\left(y_{n}\right)\right)\right)+\gamma_{n}\left(x_{n}-T\left(y_{n}\right)\right)\right\| \\
& \leq\left\|x_{n}-x_{n+1}\right\|+\alpha_{n}\left\|\rho U\left(x_{n}\right)-\mu F\left(T\left(y_{n}\right)\right)\right\|+\gamma_{n}\left\|x_{n}-T\left(y_{n}\right)\right\|,
\end{aligned}
$$

and therefore

$$
\left\|x_{n}-T\left(y_{n}\right)\right\| \leq \frac{1}{1-\gamma_{n}}\left\|x_{n}-x_{n+1}\right\|+\frac{\alpha_{n}}{1-\gamma_{n}}\left\|\rho U\left(x_{n}\right)-\mu F\left(T\left(y_{n}\right)\right)\right\| .
$$

Since $\lim _{n \rightarrow \infty}\left\|x_{n+1}-x_{n}\right\|=0, \alpha_{n} \rightarrow 0$, we obtain

$$
\lim _{n \rightarrow \infty}\left\|x_{n}-T\left(y_{n}\right)\right\|=0
$$


Since $T\left(x_{n}\right) \in C$, we have

$$
\begin{aligned}
\left\|x_{n}-T\left(x_{n}\right)\right\| \leq & \left\|x_{n}-x_{n+1}\right\|+\left\|x_{n+1}-T\left(x_{n}\right)\right\| \\
= & \left\|x_{n}-x_{n+1}\right\|+\left\|P_{C}\left[V_{n}\right]-P_{C}\left[T\left(x_{n}\right)\right]\right\| \\
\leq & \left\|x_{n}-x_{n+1}\right\|+\| \alpha_{n}\left(\rho U\left(x_{n}\right)-\mu F\left(T\left(y_{n}\right)\right)\right) \\
& \quad+\gamma_{n}\left(x_{n}-T\left(y_{n}\right)\right)+T\left(y_{n}\right)-T\left(x_{n}\right) \| \\
\leq & \left\|x_{n}-x_{n+1}\right\|+\alpha_{n}\left\|\rho U\left(x_{n}\right)-\mu F\left(T\left(y_{n}\right)\right)\right\|+\gamma_{n}\left\|x_{n}-T\left(y_{n}\right)\right\|+\left\|y_{n}-x_{n}\right\| \\
\leq & \left\|x_{n}-x_{n+1}\right\|+\alpha_{n}\left\|\rho U\left(x_{n}\right)-\mu F\left(T\left(y_{n}\right)\right)\right\|+\gamma_{n}\left\|x_{n}-T\left(y_{n}\right)\right\| \\
& +\left\|\beta_{n} S x_{n}+\left(1-\beta_{n}\right) v_{n}-x_{n}\right\| \\
\leq & \left\|x_{n}-x_{n+1}\right\|+\alpha_{n}\left\|\rho U\left(x_{n}\right)-\mu F\left(T\left(y_{n}\right)\right)\right\|+\gamma_{n}\left\|x_{n}-T\left(y_{n}\right)\right\| \\
& +\beta_{n}\left\|S x_{n}-x_{n}\right\|+\left(1-\beta_{n}\right)\left\|v_{n}-x_{n}\right\| .
\end{aligned}
$$

Since $\lim _{n \rightarrow \infty}\left\|x_{n+1}-x_{n}\right\|=0, \gamma_{n} \rightarrow 0, \alpha_{n} \rightarrow 0, \beta_{n} \rightarrow 0, \lim _{n \rightarrow \infty}\left\|x_{n}-T\left(y_{n}\right)\right\|=0$, $\left\|\rho U\left(x_{n}\right)-\mu F\left(T\left(y_{n}\right)\right)\right\|$, and $\left\|S x_{n}-x_{n}\right\|$ are bounded and $\lim _{n \rightarrow \infty}\left\|x_{n}-v_{n}\right\|=0$, we obtain

$$
\lim _{n \rightarrow \infty}\left\|x_{n}-T\left(x_{n}\right)\right\|=0
$$

Since $\left\{x_{n}\right\}$ is bounded, without loss of generality we can assume that $x_{n} \rightarrow x^{*} \in C$. It follows from Lemma 2.3 that $x^{*} \in F(T)$. Therefore $\omega_{\omega}\left(x_{n}\right) \subset F(T)$.

Theorem 3.1 The sequence $\left\{x_{n}\right\}$ generated by Algorithm 3.1 converges strongly to $z$, which is the unique solution of the variational inequality

$$
\langle\rho U(z)-\mu F(z), x-z\rangle \leq 0, \quad \forall x \in \mathscr{F}=F(T) \cap V I\left(C, B_{m}\right) \cap \operatorname{GMEP}(F, \varphi, D) .
$$

Proof Since $\left\{x_{n}\right\}$ is bounded, $x_{n} \rightarrow w$, and from Lemma 3.2, we have $w \in F(T)$. Next, we show that $w \in \operatorname{GMEP}(F, \varphi, D)$. Since $u_{n}=T_{r_{n}}\left(x_{n}-r_{n} D x_{n}\right)$, we have

$$
F\left(u_{n}, y\right)+\varphi(y)-\varphi\left(u_{n}\right)+\left\langle D x_{n}, y-u_{n}\right\rangle+\frac{1}{r_{n}}\left\langle y-u_{n}, u_{n}-x_{n}\right\rangle \geq 0, \quad \forall y \in C .
$$

It follows from the monotonicity of $F$ that

$$
\varphi(y)-\varphi\left(u_{n}\right)+\left\langle D x_{n}, y-u_{n}\right\rangle+\frac{1}{r_{n}}\left\langle y-u_{n}, u_{n}-x_{n}\right\rangle \geq F\left(y, u_{n}\right), \quad \forall y \in C,
$$

and

$$
\varphi(y)-\varphi\left(u_{n_{k}}\right)+\left\langle D x_{n_{k}}, y-u_{n_{k}}\right\rangle+\left\langle y-u_{n_{k}}, \frac{u_{n_{k}}-x_{n_{k}}}{r_{n_{k}}}\right\rangle \geq F\left(y, u_{n_{k}}\right), \quad \forall y \in C .
$$

Since $\lim _{n \rightarrow \infty}\left\|u_{n}-x_{n}\right\|=0$ and $x_{n} \rightarrow w$, it is easy to observe that $u_{n_{k}} \rightarrow w$. For any $0<t \leq 1$ and $y \in C$, let $y_{t}=t y+(1-t) w$, and we have $y_{t} \in C$. Then from (3.22), we obtain 


$$
\begin{aligned}
\left\langle D y_{t}, y_{t}-u_{n_{k}}\right\rangle \geq & \varphi\left(u_{n_{k}}\right)-\varphi\left(y_{t}\right)+\left\langle D y_{t}, y_{t}-u_{n_{k}}\right\rangle \\
& -\left\langle D x_{n_{k}}, y_{t}-u_{n_{k}}\right\rangle-\left\langle y_{t}-u_{n_{k}}, \frac{u_{n_{k}}-x_{n_{k}}}{r_{n_{k}}}\right\rangle+F\left(y_{t}, u_{n_{k}}\right) \\
= & \varphi\left(u_{n_{k}}\right)-\varphi\left(y_{t}\right)+\left\langle D y_{t}-D u_{n_{k}}, y_{t}-u_{n_{k}}\right\rangle+\left\langle D u_{n_{k}}-D x_{n_{k}}, y_{t}-u_{n_{k}}\right\rangle \\
& -\left\langle y_{t}-u_{n_{k}}, \frac{u_{n_{k}}-x_{n_{k}}}{r_{n_{k}}}\right\rangle+F\left(y_{t}, u_{n_{k}}\right) .
\end{aligned}
$$

Since $D$ is Lipschitz continuous and $\lim _{n \rightarrow \infty}\left\|u_{n}-x_{n}\right\|=0$, we obtain $\lim _{n \rightarrow \infty} \| D u_{n_{k}}-$ $D x_{n_{k}} \|=0$. From the monotonicity of $D$, the weakly lower semicontinuity of $\varphi$, and $u_{n_{k}} \rightarrow w$, it follows from (3.23) that

$$
\left\langle D y_{t}, y_{t}-w\right\rangle \geq \varphi(w)-\varphi\left(y_{t}\right)+F\left(y_{t}, w\right)
$$

Hence, from assumptions $\left(\mathrm{A}_{1}\right)-\left(\mathrm{A}_{4}\right)$ and (3.24), we have

$$
\begin{aligned}
0 & =F\left(y_{t}, y_{t}\right)+\varphi\left(y_{t}\right)-\varphi\left(y_{t}\right) \leq t F\left(y_{t}, y\right)+(1-t) F\left(y_{t}, w\right)+t \varphi(y)+(1-t) \varphi(w)-\varphi\left(y_{t}\right) \\
& =t\left[F\left(y_{t}, y\right)+\varphi(y)-\varphi\left(y_{t}\right)\right]+(1-t)\left[F\left(y_{t}, w\right)+\varphi(w)-\varphi\left(y_{t}\right)\right] \\
& \leq t\left[F\left(y_{t}, y\right)+\varphi(y)-\varphi\left(y_{t}\right)\right]+(1-t) t\left\langle D y_{t}, y-w\right\rangle,
\end{aligned}
$$

which implies that $F\left(y_{t}, y\right)+\varphi(y)-\varphi\left(y_{t}\right)+(1-t)\left\langle D y_{t}, y-w\right\rangle \geq 0$. Letting $t \rightarrow 0_{+}$, we have

$$
F(w, y)+\varphi(y)-\varphi(w)+\langle D w, y-w\rangle \geq 0, \quad \forall y \in C,
$$

which implies that $w \in \operatorname{GMEP}(F, \varphi, D)$. Furthermore, we show that $w \in V I\left(C, B_{m}\right)$. Define a mapping $J: C \rightarrow C$ by

$$
J x=\sum_{m=1}^{r} \eta^{m} P_{C}\left(I-\mu_{m} B_{m}\right) x, \quad \forall x \in C,
$$

where $\eta^{m}=\lim _{n \rightarrow \infty} \eta_{n}^{m}$. From Lemma 2.7, we see that $J$ is nonexpansive such that

$$
F(J)=\bigcap_{m=1}^{r} F\left(P_{C}\left(I-\mu_{m} B_{m}\right)\right)=\bigcap_{m=1}^{r} V I\left(C, B_{m}\right) .
$$

Note that

$$
\begin{aligned}
\left\|u_{n}-J u_{n}\right\| & \leq\left\|u_{n}-z_{n}\right\|+\left\|z_{n}-J u_{n}\right\| \\
& \leq\left\|u_{n}-z_{n}\right\|+\left\|\sum_{m=1}^{r} \eta_{n}^{m} P_{C}\left(I-\mu_{m} B_{m}\right) u_{n}-\sum_{m=1}^{r} \eta^{m} P_{C}\left(I-\mu_{m} B_{m}\right) u_{n}\right\| \\
& \leq\left\|u_{n}-z_{n}\right\|+M \cdot \sum_{m=1}^{r}\left|\eta_{n}^{m}-\eta^{m}\right| .
\end{aligned}
$$

In view of restriction (f), we find from (3.18) that

$$
\lim _{n \rightarrow \infty}\left\|u_{n}-J u_{n}\right\|=0
$$


It follows from Lemma 2.3 that $w \in F(J)=\bigcap_{m=1}^{r} V I\left(C, B_{m}\right)$. Thus, we have $w \in \mathscr{F}=F(T) \cap$ $V I\left(C, B_{m}\right) \cap \operatorname{GMEP}(F, \varphi, D)$.

Observe that the constants satisfy $0<\rho \tau<\nu$ and

$$
\begin{aligned}
\kappa \geq \eta & \Leftrightarrow \quad \kappa^{2} \geq \eta^{2} \\
& \Leftrightarrow \quad 1-2 \mu \eta+\mu^{2} \kappa^{2} \geq 1-2 \mu \eta+\mu^{2} \eta^{2} \\
& \Leftrightarrow \quad \sqrt{1-\mu\left(2 \eta-\mu \kappa^{2}\right)} \geq 1-\mu \eta \\
& \Leftrightarrow \quad \mu \eta \geq 1-\sqrt{1-\mu\left(2 \eta-\mu \kappa^{2}\right)} \\
& \Leftrightarrow \quad \mu \eta \geq v,
\end{aligned}
$$

therefore, from Lemma 2.4 , the operator $\mu F-\rho U$ is $\mu \eta-\rho \tau$-strongly monotone, and we get the uniqueness of the solution of the variation inequality (3.21) and denote it by $z \in \mathscr{F}=F(T) \cap \operatorname{VI}\left(C, B_{m}\right) \cap \operatorname{GMEP}(F, \varphi, D)$.

Next, we claim that $\lim \sup _{n \rightarrow \infty}\left\langle\rho U(z)-\mu F(z), x_{n}-z\right\rangle \leq 0$. Since $\left\{x_{n}\right\}$ is bounded, there exists a subsequence $\left\{x_{n_{k}}\right\}$ of $\left\{x_{n}\right\}$ such that

$$
\begin{aligned}
\limsup _{n \rightarrow \infty}\left\langle\rho U(z)-\mu F(z), x_{n}-z\right\rangle & =\limsup _{n \rightarrow \infty}\left\langle\rho U(z)-\mu F(z), x_{n_{k}}-z\right\rangle \\
& =\langle\rho U(z)-\mu F(z), w-z\rangle \leq 0 .
\end{aligned}
$$

Next, we show that $x_{n} \rightarrow z$. We have

$$
\begin{aligned}
\left\|x_{n+1}-z\right\|^{2} & \left\langle P_{C}\left[V_{n}\right]-z, x_{n+1}-z\right\rangle \\
= & \left\langle P_{C}\left[V_{n}\right]-V_{n}, P_{C}\left[V_{n}\right]-z\right\rangle+\left\langle V_{n}-z, x_{n+1}-z\right\rangle \\
\leq & \left\langle\alpha_{n}\left(\rho U\left(x_{n}\right)-\mu F(z)\right)+\gamma_{n}\left(x_{n}-z\right)\right. \\
& \left.+\left(1-\gamma_{n}\right)\left[\left(I-\frac{\alpha_{n} \mu}{1-\gamma_{n}} F\right) T\left(y_{n}\right)-\left(I-\frac{\alpha_{n} \mu}{1-\gamma_{n}} F\right) T(z)\right], x_{n+1}-z\right\rangle \\
= & \left\langle\alpha_{n} \rho\left(U\left(x_{n}\right)-U(z)\right), x_{n+1}-z\right\rangle+\alpha_{n}\left\langle\rho U(z)-\mu F(z), x_{n+1}-z\right\rangle \\
& +\gamma_{n}\left\langle x_{n}-z, x_{n+1}-z\right\rangle \\
& \left.\left.+\left(1-\gamma_{n}\right)\right\rangle\left(I-\frac{\alpha_{n} \mu}{1-\gamma_{n}} F\right) T\left(y_{n}\right)-\left(I-\frac{\alpha_{n} \mu}{1-\gamma_{n}} F\right) T(z), x_{n+1}-z\right\rangle \\
\leq & \left(\gamma_{n}+\alpha_{n} \rho \tau\right)\left\|x_{n}-z\right\|\left\|x_{n+1}-z\right\|+\alpha_{n}\left\langle\rho U(z)-\mu F(z), x_{n+1}-z\right\rangle \\
& +\left(1-\gamma_{n}-\alpha_{n} \nu\right)\left\|y_{n}-z\right\|\left\|x_{n+1}-z\right\| \\
\leq & \left(\gamma_{n}+\alpha_{n} \rho \tau\right)\left\|x_{n}-z\right\|\left\|x_{n+1}-z\right\|+\alpha_{n}\left\langle\rho U(z)-\mu F(z), x_{n+1}-z\right\rangle \\
& +\left(1-\gamma_{n}-\alpha_{n} \nu\right)\left\{\beta_{n}\left\|S x_{n}-S z\right\|+\beta_{n}\|S z-z\|+\left(1-\beta_{n}\right)\left\|v_{n}-z\right\|\right\} \\
& \times\left\|x_{n+1}-z\right\| \\
\leq & \left(\gamma_{n}+\alpha_{n} \rho \tau\right)\left\|x_{n}-z\right\|\left\|x_{n+1}-z\right\|+\alpha_{n}\left\langle\rho U(z)-\mu F(z), x_{n+1}-z\right\rangle \\
& (1-z)
\end{aligned}
$$




$$
\begin{aligned}
& +\left(1-\gamma_{n}-\alpha_{n} \nu\right)\left\{\beta_{n}\left\|x_{n}-z\right\|+\beta_{n}\|S z-z\|+\left(1-\beta_{n}\right)\left\|x_{n}-z\right\|\right\}\left\|x_{n+1}-z\right\| \\
= & \left(1-\alpha_{n}(\nu-\rho \tau)\right)\left\|x_{n}-z\right\|\left\|x_{n+1}-z\right\|+\alpha_{n}\left\langle\rho U(z)-\mu F(z), x_{n+1}-z\right\rangle \\
& +\left(1-\gamma_{n}-\alpha_{n} \nu\right) \beta_{n}\|S z-z\|\left\|x_{n+1}-z\right\| \\
\leq & \frac{1-\alpha_{n}(\nu-\rho \tau)}{2}\left(\left\|x_{n}-z\right\|^{2}+\left\|x_{n+1}-z\right\|^{2}\right)+\alpha_{n}\left\langle\rho U(z)-\mu F(z), x_{n+1}-z\right\rangle \\
& +\left(1-\gamma_{n}-\alpha_{n} \nu\right) \beta_{n}\|S z-z\|\left\|x_{n+1}-z\right\|,
\end{aligned}
$$

which implies that

$$
\begin{aligned}
\| x_{n+1} & -z \|^{2} \\
\leq & \frac{1-\alpha_{n}(v-\rho \tau)}{1+\alpha_{n}(v-\rho \tau)}\left\|x_{n}-z\right\|^{2}+\frac{2 \alpha_{n}}{1+\alpha_{n}(v-\rho \tau)}\left\langle\rho U(z)-\mu F(z), x_{n+1}-z\right\rangle \\
& +\frac{2\left(1-\gamma_{n}-\alpha_{n} v\right) \beta_{n}}{1+\alpha_{n}(v-\rho \tau)}\|S z-z\|\left\|x_{n+1}-z\right\| \\
\leq & \left(1-\alpha_{n}(v-\rho \tau)\right)\left\|x_{n}-z\right\|^{2}+\frac{2 \alpha_{n}(v-\rho \tau)}{1+\alpha_{n}(v-\rho \tau)} \\
& \times\left\{\frac{1}{v-\rho \tau}\left\langle\rho U(z)-\mu F(z), x_{n+1}-z\right\rangle+\frac{\left(1-\gamma_{n}-\alpha_{n} v\right) \beta_{n}}{\alpha_{n}(v-\rho \tau)}\|S z-z\|\left\|x_{n+1}-z\right\|\right\} .
\end{aligned}
$$

It follows from Lemma 2.6 that $x_{n} \rightarrow z$. This completes the proof.

\section{Applications}

In this section, we obtain the following results by using a special case of the proposed method for example.

Putting $D=\varphi=0, B_{m}=0$ for each $m$, and $\delta_{n}=0$ in Algorithm 3.1, we obtain the following result, which can be viewed as an extension and improvement of the method of Bnouhachem et al. [22] for finding the approximate element of the common set of solutions of equilibrium problem and a hierarchical fixed point problem.

Theorem 4.1 Let $C$ be a nonempty, closed, and convex subset of a real Hilbert space $H$. Let $F: C \times C \rightarrow R$ be a bifunction that satisfy condition $\left(\mathrm{A}_{1}\right)-\left(\mathrm{A}_{4}\right)$, and let $S, T: C \rightarrow C$ be nonexpansive mappings such that $F(T) \cap E P(F) \neq \emptyset$. Let $F: C \rightarrow C$ be a $\kappa$-Lipschitzian mapping and $\eta$-strongly monotone, and let $U: C \rightarrow C$ be a $\tau$-Lipschitzian mapping. For an arbitrarily given $x_{0} \in C$, let the iterative sequences $\left\{u_{n}\right\},\left\{x_{n}\right\}$, and $\left\{y_{n}\right\}$ be generated by

$$
\left\{\begin{array}{l}
F\left(u_{n}, y\right)+\frac{1}{r_{n}}\left\langle y-u_{n}, u_{n}-x_{n}\right\rangle \geq 0, \quad \forall y \in C ; \\
y_{n}=\beta_{n} S x_{n}+\left(1-\beta_{n}\right) u_{n} \\
x_{n+1}=P_{C}\left[\alpha_{n} \rho U\left(x_{n}\right)+\gamma_{n} x_{n}+\left(\left(1-\gamma_{n}\right) I-\alpha_{n} \mu F\right) T\left(y_{n}\right)\right], \quad \forall n \geq 0 .
\end{array}\right.
$$

Suppose that the parameters satisfy $0<\mu<\frac{2 \eta}{\kappa^{2}}, 0 \leq \rho \tau<v$, where $v=1-\sqrt{1-\mu\left(2 \eta-\mu \kappa^{2}\right)}$. Also $\left\{\gamma_{n}\right\},\left\{\alpha_{n}\right\},\left\{\beta_{n}\right\}$, and $\left\{r_{n}\right\}$ are sequences in $(0,1)$ satisfying the following conditions:

(a) $\lim _{n \rightarrow \infty} \gamma_{n}=0, \gamma_{n}+\alpha_{n}<1$;

(b) $\lim _{n \rightarrow \infty} \alpha_{n}=0$, and $\sum_{n=1}^{\infty} \alpha_{n}=\infty$;

(c) $\lim _{n \rightarrow \infty}\left(\beta_{n} / \alpha_{n}\right)=0$;

(d) $\sum_{n=1}^{\infty}\left|\alpha_{n}-\alpha_{n-1}\right|<\infty, \sum_{n=1}^{\infty}\left|\gamma_{n}-\gamma_{n-1}\right|<\infty$, and $\sum_{n=1}^{\infty}\left|\beta_{n}-\beta_{n-1}\right|<\infty$;

(e) $\liminf _{n \rightarrow \infty} r_{n}>0$, and $\sum_{n=1}^{\infty}\left|r_{n}-r_{n-1}\right|<\infty$. 
Then the sequence $\left\{x_{n}\right\}$ converges strongly to $z$, which is the unique solution of the variational inequality:

$$
\langle\rho U(z)-\mu F(z), x-z\rangle \leq 0, \quad \forall x \in F(T) \cap E P(F) .
$$

Putting $\delta_{n}=0, m=1$ in Algorithm 3.1, we obtain the following result which can be viewed as an extension and improvement of the method of Bnouhachem and Chen [23] for finding the approximate element of the common set of solutions of variational inequalities, a generalized mixed equilibrium problem, and a hierarchical fixed point problem.

Theorem 4.2 Let $C$ be a nonempty, closed, and convex subset of a real Hilbert space H. Let $D, A: C \rightarrow H$ be $\theta$, $\alpha$-inverse strongly monotone mapping, respectively. Let $F: C \times C \rightarrow R$ satisfy $\left(\mathrm{A}_{1}\right)-\left(\mathrm{A}_{4}\right)$, and let $\varphi: C \rightarrow R$ be a proper lower semicontinuous and convex function. Let $S, T: C \rightarrow C$ be nonexpansive mappings such that $F(T) \cap \operatorname{VI}(C, A) \cap \operatorname{GMEP}(F, \varphi, D) \neq$ $\emptyset$. Let $F: C \rightarrow C$ be a $\kappa$-Lipschitzian mapping and be $\eta$-strongly monotone, and let $U: C \rightarrow$ $C$ be a $\tau$-Lipschitzian mapping. For an arbitrarily given $x_{0} \in C$, let the iterative sequences $\left\{u_{n}\right\},\left\{x_{n}\right\},\left\{y_{n}\right\}$, and $\left\{z_{n}\right\}$ be generated by

$$
\left\{\begin{array}{l}
F\left(u_{n}, y\right)+\left\langle D x_{n}, y-u_{n}\right\rangle+\varphi(y)-\varphi\left(u_{n}\right)+\frac{1}{r_{n}}\left\langle y-u_{n}, u_{n}-x_{n}\right\rangle \geq 0, \quad \forall y \in C ; \\
z_{n}=P_{C}\left[u_{n}-\lambda_{n} A u_{n}\right] ; \\
y_{n}=\beta_{n} S x_{n}+\left(1-\beta_{n}\right) z_{n} ; \\
x_{n+1}=P_{C}\left[\alpha_{n} \rho U\left(x_{n}\right)+\gamma_{n} x_{n}+\left(\left(1-\gamma_{n}\right) I-\alpha_{n} \mu F\right) T\left(y_{n}\right)\right], \quad \forall n \geq 0,
\end{array}\right.
$$

where $\lambda_{n} \in(0,2 \alpha),\left\{r_{n}\right\} \subset(2,2 \theta)$. Suppose that the parameters satisfy $0<\mu<\frac{2 \eta}{\kappa^{2}}, 0 \leq \rho \tau<$ $v$, where $v=1-\sqrt{1-\mu\left(2 \eta-\mu \kappa^{2}\right)}$. Also $\left\{\alpha_{n}\right\},\left\{\beta_{n}\right\}$, and $\left\{\gamma_{n}\right\}$ are sequences in $(0,1)$ satisfying the following conditions:

(a) $\lim _{n \rightarrow \infty} \gamma_{n}=0, \gamma_{n}+\alpha_{n}<1$;

(b) $\lim _{n \rightarrow \infty} \alpha_{n}=0$, and $\sum_{n=1}^{\infty} \alpha_{n}=\infty$;

(c) $\lim _{n \rightarrow \infty}\left(\beta_{n} / \alpha_{n}\right)=0$;

(d) $\sum_{n=1}^{\infty}\left|\alpha_{n}-\alpha_{n-1}\right|<\infty, \sum_{n=1}^{\infty}\left|\gamma_{n}-\gamma_{n-1}\right|<\infty$, and $\sum_{n=1}^{\infty}\left|\beta_{n}-\beta_{n-1}\right|<\infty$;

(e) $\liminf _{n \rightarrow \infty} r_{n}>0$, and $\sum_{n=1}^{\infty}\left|r_{n}-r_{n-1}\right|<\infty$;

(f) $\liminf _{n \rightarrow \infty} \lambda_{n}<\lim \sup _{n \rightarrow \infty} \lambda_{n}<2 \alpha$ and $\sum_{n=1}^{\infty}\left|\lambda_{n}-\lambda_{n-1}\right|<\infty$.

Then the sequence $\left\{x_{n}\right\}$ converges strongly to $z$, which is the unique solution of the variational inequality:

$$
\langle\rho U(z)-\mu F(z), x-z\rangle \leq 0, \quad \forall x \in V I(C, A) \cap \operatorname{GMEP}(F, \varphi, D) \cap F(T) .
$$

Putting $\rho=\mu=1, \beta_{n}=\delta_{n}=0, \varphi=0, U=f$ a contraction mapping, and $F=A$ a strongly positive linear bounded operator, we obtain the following theorem.

Theorem 4.3 Let $C$ be a nonempty, closed, and convex subset of a real Hilbert space $H$. Let $B_{m}: C \rightarrow H$ be $l_{m}$-inverse strongly monotone mapping for each $1 \leq m \leq r$, where $r$ is some positive integer. Let $D: C \rightarrow H$ be a $\alpha$-inverse strongly monotone mapping. Let $F: C \times$ $C \rightarrow R$ satisfy $\left(\mathrm{A}_{1}\right)-\left(\mathrm{A}_{4}\right)$. Let $T: C \rightarrow C$ be nonexpansive mappings such that $\mathscr{F}=F(T) \cap$ $V I(C, A) \cap E P \neq \emptyset$. Let $A$ be a strongly positive linear bounded operator with coefficient $\bar{\gamma}$ 
and let $f: H \rightarrow H$ be a contraction with contraction constant $h(0<h<1)$ and $0<\gamma<$ $(\bar{\gamma} / h)$. Let $\left\{x_{n}\right\},\left\{y_{n}\right\},\left\{\rho_{n}\right\}$ be sequences generated by $x_{1} \in H$ and

$$
\left\{\begin{array}{l}
F\left(y_{n}, \eta\right)+\left\langle D y_{n}, \eta-y_{n}\right\rangle+\frac{1}{r_{n}}\left\langle\eta-y_{n}, y_{n}-x_{n}\right\rangle \geq 0, \quad \forall \eta \in C \\
\rho_{n}=\sum_{m=1}^{r} \eta_{n}^{m} P_{C}\left(I-\mu_{m} B_{m}\right) y_{n} \\
x_{n+1}=\alpha_{n} \gamma f\left(x_{n}\right)+\beta_{n} x_{n}+\left(\left(1-\beta_{n}\right) I-\alpha_{n} A\right) T\left(\rho_{n}\right)
\end{array}\right.
$$

where $\mu_{m} \in\left(0,2 l_{m}\right),\{\alpha\},\{\beta\} \subset[0,1]$, and $\left\{r_{n}\right\} \subset[0, \infty]$. If the following conditions are satisfied:

(a) $\lim _{n \rightarrow \infty} \alpha_{n}=0$, and $\sum_{n=1}^{\infty} \alpha_{n}=\infty$;

(b) $\lim _{n \rightarrow \infty} \eta_{n}^{m}=\eta^{m} \in(0,1)$;

(c) $\sum_{n=1}^{\infty}\left|r_{n+1}-r_{n}\right|<\infty$;

(d) $\liminf _{n \rightarrow \infty} r_{n}>0,0<\liminf _{n \rightarrow \infty} \beta_{n} \leq \limsup _{n \rightarrow \infty} \beta_{n}<1$;

(e) $\sum_{m=1}^{r} \eta_{n}^{m}=1, \forall n \geq 1$;

then $\left\{x_{n}\right\}$ converges strongly to $q \in \mathscr{F}$, where $q=P_{\mathscr{F}}(\gamma f+(I-A)) q$.

Remark If $T=W_{n}$ in Theorem 4.3, where $W_{n}$ is the $W$-mapping of $C$ into itself which is generated by a family of nonexpansive mappings $S_{n}, S_{n-1}, \ldots, S_{1}$, and a sequence of positive numbers in $[0,1] \lambda_{n}, \lambda_{n-1}, \ldots, \lambda_{1}$, we can easily get Theorem 10 in Zhou et al. [13]. It is worth to mention two points as follows:

(1) Since we all know that $W_{n}$ mapping is nonexpansive, if $T=W_{n}$ in Theorem 4.3, then we can easily get Theorem 10 in Zhou et al. [13].

(2) A family of infinite $k_{n}$-strict pseudocontractive mappings in Theorem 10 in Zhou et al. [13] did not work, so we should omit them. Theorem 10 in Zhou et al. [13] should be corrected as follows:

Theorem 4.4 Let $C$ be a nonempty, closed, and convex subset of a real Hilbert space $H$. Let $B_{m}: C \rightarrow H$ be $l_{m}$-inverse strongly monotone mapping for each $1 \leq m \leq r$, where $r$ is some positive integer. Let $D: C \rightarrow H$ be a $\alpha$-inverse strongly monotone mapping. Let $F: C \times C \rightarrow R$ satisfy $\left(\mathrm{A}_{1}\right)-\left(\mathrm{A}_{4}\right)$. Let $\left\{\lambda_{n}\right\}_{n=1}^{\infty}$ be a sequence of positive numbers in $[0, b]$ for some $b \in(0,1)$, and let $\left\{S_{n}\right\}_{n=1}^{\infty}: C \rightarrow C$ be a family of infinitely nonexpansive mappings such that $\mathscr{F}=F(T) \cap V I(C, A) \cap E P \neq \emptyset$. Let $A$ be a strongly positive linear bounded operator with coefficient $\bar{\gamma}$ and let $f: H \rightarrow H$ be a contraction with contraction constant $h(0<h<1)$ and $0<\gamma<(\bar{\gamma} / h)$. Let $\left\{x_{n}\right\},\left\{y_{n}\right\},\left\{\rho_{n}\right\}$ be sequences generated by $x_{1} \in H$ and

$$
\left\{\begin{array}{l}
F\left(y_{n}, \eta\right)+\left\langle D y_{n}, \eta-y_{n}\right\rangle+\frac{1}{r_{n}}\left\langle\eta-y_{n}, y_{n}-x_{n}\right\rangle \geq 0, \quad \forall \eta \in C \\
\rho_{n}=\sum_{m=1}^{r} \eta_{n}^{m} P_{C}\left(I-\mu_{m} B_{m}\right) y_{n} \\
x_{n+1}=\alpha_{n} \gamma f\left(x_{n}\right)+\beta_{n} x_{n}+\left(\left(1-\beta_{n}\right) I-\alpha_{n} A\right) W_{n} \rho_{n}
\end{array}\right.
$$

where $\mu_{m} \in\left(0,2 l_{m}\right),\{\alpha\},\{\beta\} \subset[0,1]$, and $\left\{r_{n}\right\} \subset[0, \infty]$. If the following conditions are satisfied:

(a) $\lim _{n \rightarrow \infty} \alpha_{n}=0$, and $\sum_{n=1}^{\infty} \alpha_{n}=\infty$;

(b) $\lim _{n \rightarrow \infty} \eta_{n}^{m}=\eta^{m} \in(0,1)$;

(c) $\sum_{n=1}^{\infty}\left|r_{n+1}-r_{n}\right|<\infty$;

(d) $\liminf _{n \rightarrow \infty} r_{n}>0,0<\liminf _{n \rightarrow \infty} \beta_{n} \leq \limsup _{n \rightarrow \infty} \beta_{n}<1$;

(e) $\sum_{m=1}^{r} \eta_{n}^{m}=1, \forall n \geq 1$;

then $\left\{x_{n}\right\}$ converges strongly to $q \in \mathscr{F}$, where $q=P_{\mathscr{F}}(\gamma f+(I-A)) q$. 


\section{Competing interests}

The authors declare that they have no competing interest.

\section{Authors' contributions}

All authors contributed equally and significantly in writing this article. All authors read and approved the final manuscript.

\section{Acknowledgements}

All the authors were partially supported by the National Science Foundation of China (11071169) and Ph.D. Program

Foundation of the Ministry of Education of China (20123127110002).

\section{Received: 2 February 2015 Accepted: 21 April 2015 Published online: 09 May 2015}

\section{References}

1. Peng, JW, Yao, JC: A new hybrid-extragradient method for generalized mixed equilibrium problems and fixed point problems and variational inequality problems. Taiwan. J. Math. 12(6), 1401-1432 (2008)

2. Al-Mazrooei, AE, Latif, A, Yao, JC: Solving generalized mixed equilibria, variational inequalities, and constrained convex minimization. Abstr. Appl. Anal. 2014, Article ID 587865 (2014)

3. Anasari, QH, Schaible, S, Yao, JC: The system of generalized vector equilibrium problems with applications. J. Glob. Optim. 22, 3-16 (2002)

4. Ceng, LC, Hu, HY, Wong, MM: Strong and weak convergence theorems for generalized mixed equilibrium problem with perturbation and fixed pointed problem of infinitely many nonexpansive mappings. Taiwan. J. Math. 15(3), 1341-1367 (2011)

5. Ceng, LC, Guu, SM, Yao, JC: Hybrid iterative method for finding common solutions of generalized mixed equilibrium and fixed point problems. Fixed Point Theory Appl. 2012, Article ID 92 (2012)

6. Ceng, LC, Ansari, QH, Schaible, S, Yao, JC: Iterative methods for generalized equilibrium problems, systems of generalized equilibrium problems and fixed point problems for nonexpansive mapping in Hilbert spaces. Fixed Point Theory 12(2), 293-308 (2011)

7. Ceng, LC, Yao, JC: A hybrid iterative scheme for mixed equilibrium problems and fixed point problems. J. Comput. Appl. Math. 214, 186-201 (2008)

8. Takahashi, S, Takahashi, W: Strong convergence theorem for a generalized equilibrium problem and a nonexpansive mapping in a Hilbert spaces. Nonlinear Anal. 69, 1025-1033 (2008)

9. Chang, SS, Joseph Lee, HW, Chan, CK: A new method for solving equilibrium problem fixed point problem and variational inequality problem with application to optimization. Nonlinear Anal. 70, 3307-3319 (2009)

10. Qin, X, Shang, M, Su, Y: A general iterative method for equilibrium problem and fixed point problem in Hilbert space. Nonlinear Anal. 69, 3897-3909 (2008)

11. Takahashi, W, Toyoda, M: Weak convergence theorems for nonexpansive mappings and monotone mapping. J. Optim. Theory Appl. 118(2), 417-428 (2003)

12. Yao, Y, Yao, JC: On modified iterative method for nonexpansive mappings and monotone mapping. Appl. Math. Comput. 186(2), 1551-1558 (2007)

13. Zhou, YY, Cao, JT, Wang, YL: Convergence theorem for equilibrium and variational inequality problems and a family of infinitely nonexpansive mappings in Hilbert space. J. Appl. Math. 2014, Article ID 232541 (2014)

14. Yao, Y, Cho, YJ, Liou, YC: Iterative algorithms for hierarchical fixed points problems and variational inequalities. Math Comput. Model. 52(9-10), 1697-1705 (2010)

15. Mainge, PE, Moudafi, A: Strong convergence of an iterative method for hierarchical fixed-point problems. Pac. J. Optim. 3(3), 529-538 (2007)

16. Moudafi, A: Krasnoselski-Mann iteration for hierarchical fixed-point problems. Inverse Probl. 23(4), 1635-1640 (2007)

17. Cianciaruso, F, Marino, G, Muglia, L, Yao, Y: On a two-step algorithm for hierarchical fixed point problems and variational inequalities. J. Inequal. Appl. 2009, Article ID 208692 (2009)

18. Gu, G, Wang, S, Cho, YJ: Strong convergence algorithms for hierarchical fixed points problems and variational inequalities. J. Appl. Math. 2011, Article ID 164978 (2011)

19. Marino, G, Xu, HK: Explicit hierarchical fixed point approach to variational inequalities. J. Optim. Theory Appl. 149(1), 61-78 (2011)

20. Ceng, LC, Ansari, QH, Yao, JC: Some iterative methods for finding fixed points and for solving constrained convex minimization problems. Nonlinear Anal. 74, 5286-5302 (2011)

21. Ceng, LC, Al-Mezel, SA, Latif, A: Hybrid viscosity approaches to general systems of variational inequalities with hierarchical fixed point problem constrains in Banach space. Abstr. Appl. Anal. 2014, Article ID 945985 (2014)

22. Bnouhachem, A, Al-Homidan, S, Ansari, QH: An iterative method for common solutions of equilibrium problems and hierarchical fixed point problems. Fixed Point Theory Appl. 2014, Article ID 194 (2014)

23. Bnouhachem, A, Chen, Y: An iterative method for a common solution of generalized mixed equilibrium problems, variational inequalities, and hierarchical fixed point problems. Fixed Point Theory Appl. 2014, Article ID 155 (2014)

24. Latif, A, Ceng, LC, Ansari, QH: Multi-step hybrid viscosity method for systems of variational inequalities defined over sets of solutions of an equilibrium problem and fixed point problems. Fixed Point Theory Appl. 2012, Article ID 186 (2012)

25. Ceng, LC, Ansari, QH, Schaible, S: Hybrid extragradient-like methods for generalized mixed equilibrium problems, systems of generalized equilibrium problems and optimization problems. J. Glob. Optim. 53(1), 69-96 (2012)

26. Zeng, LC, Ansari, QH, Al-Homidan, S: Hybrid proximal-type algorithms for generalized equilibrium problems, maximal monotone operators, and relatively nonexpansive mapping. Fixed Point Theory Appl. 2011, Article ID 973028 (2011)

27. Ceng, LC, Ansari, QH, Yao, JC: Hybrid pseudoviscosity approximation schemes for equilibrium problems and fixed point problems of infinitely many nonexpansive mappings. Nonlinear Anal. Hybrid Syst. 4(4), 743-754 (2010)

28. Geobel, K, Kirk, WA: Topics in Metric Fixed Point Theory. Stud. Adv. Math., vol. 28. Cambridge University Press, Cambridge (1990)

29. Suzuki, N: Moudafi's viscosity approximations with Meir-Keeler contractions. J. Math. Anal. Appl. 325, 342-352 (2007) 
30. Liu, LS: Iterative process with errors for nonlinear strongly accretive mapping in Banach spaces. J. Math. Anal. Appl. 194, 114-125 (1995)

31. Cho, SY, Kang, SM: Approximation of common solutions of variational inequalities via strict pseudocontractions. Acta Math. Sci. 32(4), 1601-1618 (2012)

Submit your manuscript to a SpringerOpen ${ }^{\circ}$ journal and benefit from:

- Convenient online submission

Rigorous peer review

- Immediate publication on acceptance

- Open access: articles freely available online

- High visibility within the field

- Retaining the copyright to your article

Submit your next manuscript at $\boldsymbol{s p r i n g e r o p e n . c o m ~}$ 